\title{
Finally, Bulk Typing of Bacterial Species down to Strain Level using ON-rep-seq
}

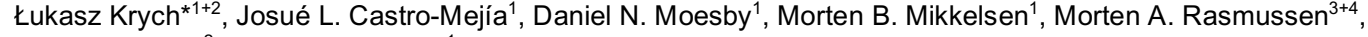
Maciej Sykulski ${ }^{2}$, Dennis S. Nielsen ${ }^{1}$

${ }^{1}$ Food Microbiology \& Fermentation, Department of Food Science, University of Copenhagen, 1958 Frederiksberg C, Denmark; ${ }^{2}$ GenXone Sp. Z O.O., 60-476 Poznań, Poland; ${ }^{3}$ Chemometrics \& Analytical Technology, Department of Food Science, University of Copenhagen, 1958 Frederiksberg C, Denmark; ${ }^{4}$ COPSAC, Copenhagen Prospective Studies on Asthma in Childhood, Herlev and Gentofte Hospital, University of Copenhagen, Copenhagen, Denmark
}

\section{Abstract}

Despite the massive developments within culture-independent methods for detection and quantification of microorganisms during the last decade, culture-based methods remain a cornerstone in microbiology. We have developed a new method for bacterial DNA enrichment and tagmentation allowing fast $(<24 \mathrm{~h})$ and cost-effective species level identification and strain level differentiation using the MinION portable sequencing platform (ON-repseq). DNA library preparation takes less than $5 \mathrm{~h}$ and ensures highly reproducible distribution of reads that can be used to generate strain level specific read length counts profiles (LCp). We have developed a pipeline that by correcting the random error of reads within peaks of LCp generates a set ( $\sim 10$ contigs per sample; $300 \mathrm{bp}-3 \mathrm{~Kb})$ of high quality (>99\%) consensus reads. Whereas, the information from high quality reads is used to retrieve species level taxonomy, comparison of LCp allows for strain level differentiation. With benchmarked 288 isolates identified on a single flow cell and a theoretical throughput to evaluate over 1000 isolates, our method allows for detailed bacterial identification for less than $2 \$$ per sample at very high speed. 


\section{Introduction}

Culture dependent methods remain indispensable in detailed identification of bacteria. Yet, successful typing of bacteria down to species/strain level remains not fully resolved ${ }^{1}$. Several promising technologies and methodologies for solving the problem have been proposed but with a variable success. Generally, fast and cost-effective methods are not accurate enough, while those that are more accurate are also more laborious and/or expensive. Methods based on 16S rRNA gene sequencing are amongst the most universal, yet species level resolution cannot always be reached ${ }^{2}$. More complex molecular tools that are able to reach strain level resolution such as PFGE, Rep-PCR, MLST or MALDI-TOF MS are hampered by one or several drawbacks that include low speed/throughput, limited databases, no taxonomic information, laborious procedure or high equipment cost $3,4,5$.

The present gold standard for strain level bacterial identification is full genome sequencing. Optimally this approach combines information from highthroughput, short, good quality reads with lower throughput, poor quality but long reads ${ }^{6}$. However, this approach is far from being cost effective, and the data analysis and interpretation is far from trivial $7,8,9$.

The portable DNA sequencing platform MinION by Oxford Nanopore Technologies (ONT) offers an attractive tool with a potential to tackle the task of species/strain level identification ${ }^{10}$. Unfortunately, ONT still deals with two critical problems: relatively high error rate at the base level and lower throughput compared to technologies offered by e.g. Illumina ${ }^{10}$. We propose a DNA enrichment method that to a large extent have solved both these pitfalls by combining an optimized version of repetitive extragenic palindromic PCR (Rep-PCR) with a consecutive dual-stage Rep-PCR-2 step during which sample specific barcodes are incorporated.

Repetitive extragenic palindromic sequences in bacterial genomes were first described in the genomes of E. coli and Salmonella in 1984 by M.J. Stern ${ }^{11}$. A decade later J. Versalovic used interspersed repetitive sequences as a binding site for primers developing Rep-PCR ${ }^{12}$. Amplicons varying in length (from few dozens base pairs (bp) to few kilo base pairs (Kbp)) separated with electrophoresis create a genomic fingerprint that has been proven many times to have species and in some set-ups also strain level 
discriminative resolution of bacteria ${ }^{13}$. Only five years later Rep-PCR was described as one of the most reproducible and commonly used method for species and strain level discernment ${ }^{14}$, and numerous applications of the method have been reported in many fields including food processing, food safety, environmental microbiology, and medicine 15,16,17,18,19. Despite the immense progress in DNA sequencing technologies Rep-PCR is still a commonly used technique in many research groups mainly due to the low cost of the analysis and basic laboratory equipment needed ${ }^{20}$. However, the low running costs comes with a price of highly laborious and time-consuming procedures involving 3-5h PCR, 3-5h electrophoresis, and complicated, tedious and potentially error prone fingerprint data analysis. Additionally, RepPCR only allows for bacterial discrimination but not direct identification ${ }^{21}$.

We are presenting a new bacterial DNA enrichment method for Oxford Nanopore sequencing called ON-rep-seq. The method exploits an optimized version of Rep-PCR for reproducible amplification followed by a dual stage Rep-PCR-2 step allowing tagmentation of up to 96 samples in one reaction. Furthermore, we have developed a pipeline utilizing the information from the generated sequences at three levels: i) generation and comparison of isolate specific read length counts profiles (LCp) ii) detection of peaks in each LCp followed by within-peak correction of the random single base error iii) specieslevel taxonomy assignment using corrected consensus reads (Figure 1). The method has been tested on 38 different bacterial species and three strain level groups successfully identifying all bacteria down to the species level and discriminating strains with a sensitivity that is at least similar to a Whole Genome Sequencing (WGS) based approach. 


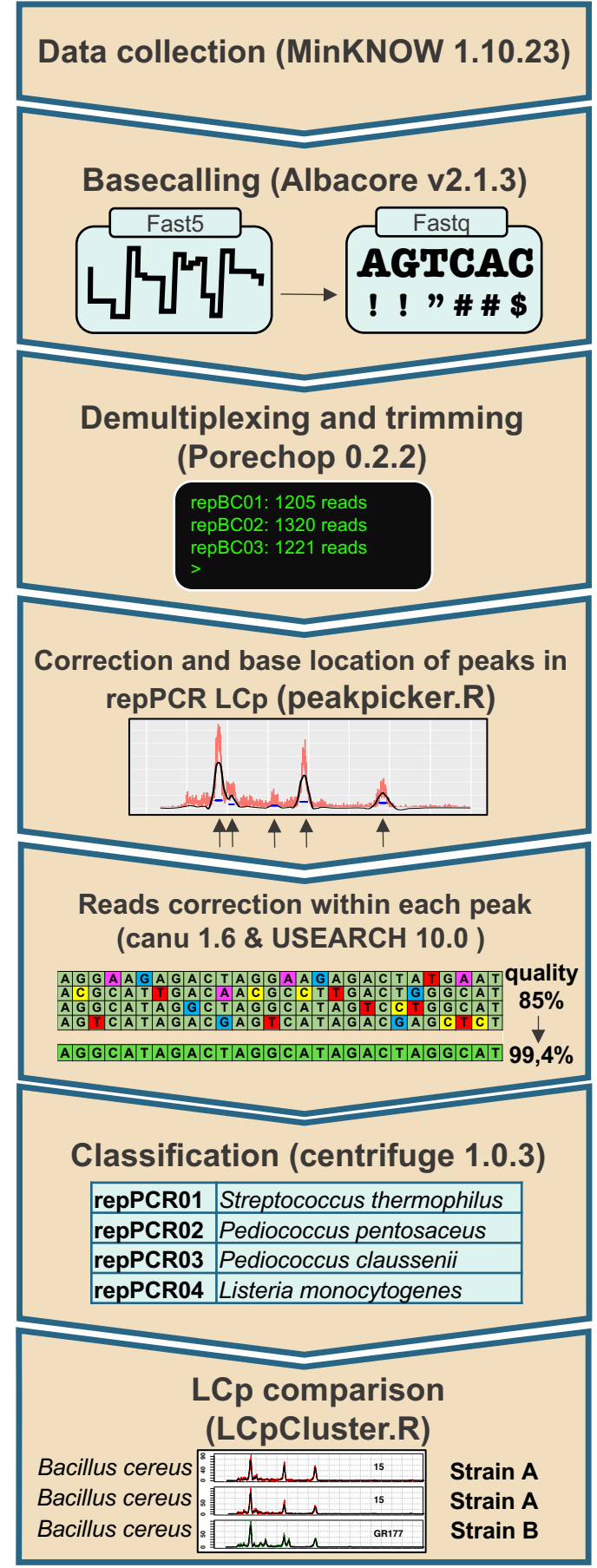

Figure 1. ON-rep-seq pipeline overview

The schema describing pipeline that allows processing of the raw Oxford Nanopore Technology based rep-PCR amplicon sequencing (ON-rep-seq) data. After initial besecalling, demultiplexing (separating according to barcodes) the fastq files are used to generate read length counts profiles (LCp) based on sequences length distribution. Subsequently, reads within each peak are clustered with USEARCH, corrected with Canu, followed by Centrifuge based taxonomy classification using improved quality reads. Finally, the traces can be compared to estimate strain level relatedness between pairs of LCp. 


\section{Results}

\section{Sequencing of the Rep-PCR enriched library with MinION generates highly reproducible LCp}

Similar to Rep-PCR gel based fingerprints, sequenced Rep-PCR products can be transformed into read length counts profiles (LCp) being a function of reads length and abundance. The shape and position of peaks is highly reproducible in all technical replicates across first two sequencing runs (Figure 2, Supplementary Figures 1 and 2) indicating that the barcode sequences do not affect the shape or the position of the peaks during RepPCR-2. Yet, as explained below, we observed a minor run effect in the third consecutive run resulting in shifted distribution of short/long reads.

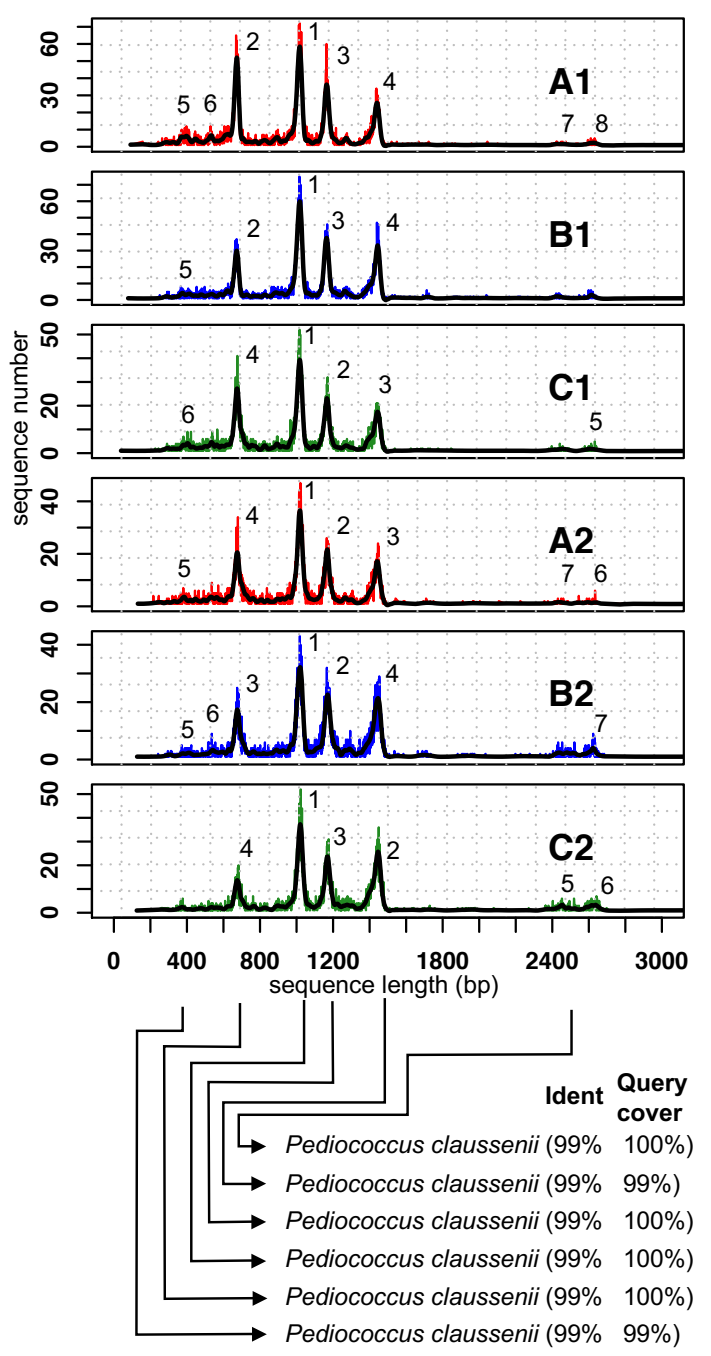

Figure 2. Pediococcus claussenii ON-rep-seq LCp Read length counts profiles (LCp) generated from Oxford Nanopore Technology based rep-PCR amplicon sequencing (ON-rep-seq) of Pediococcus claussenii. All technical replicates of $P$. clausenni profiles show high level of similarity across three consecutive sequencing runs (red, blue and green for run A, B and C respectively) and two technical replicates in each run. The retrieved sequences matching the length of a corresponding peak were subjected for correction using Canu and consensus sequences were verified using blastn. For all profiles six to eight high quality reads could be generated, each with $>99 \%$ similarity to the reference genome of $P$. clausenni. The numbers above each peak indicates the peak detection sensitivity with 1 being the most evident. The minimum number of reads within the peak needed for reads correction is 50 . 


\section{Reads correction within individual peaks provides a set of high quality} consensus sequences per isolate allowing detailed identification

A single band on a gel (or peak in LCp) of a Rep-PCR profile will contain mainly representatives of the same amplicon what would allow for base accuracy correction using tools such as e.g. Canu ${ }^{22}$. With that assumption, we have developed a pipeline operating in three steps: strain specific LCp generation and comparison, within peak reads correction, and peak's consensus sequence annotation. The pipeline generated on average 10 high quality consensus reads for each isolate $(\max =26, \min =3, \operatorname{SD}=4)$ with mean length of $1 \mathrm{Kbp}(\max =3.6 \mathrm{Kbp}, \min =0.3 \mathrm{Kbp}, \mathrm{SD}=0.6 \mathrm{Kbp})$. The number of reads used for correction within a peak (cluster size) varied from 50 to $2400($ mean $=254, S D=246)$.

Subjecting the set of corrected reads for each sample to centrifuge classifier allowed for unambiguous annotation of all bacteria down to the species and subspecies level (Table 1). The average sequence similarity of corrected reads from strain validated with Illumina sequencing ( $S$. enterica serovar Typhimurium C5) reached 99,4\% (BLAST; $\min =98.3 \%, \max =100 \%, \mathrm{SD}=$ $0.5 \%$ ). Among the isolates tested are for example Lactobacillus casei and Lactobacillus paracasei subsp. paracasei known to be indistinguishable based on $16 \mathrm{~S}$ rRNA gene sequence comparison or Lactococcus lactis subsp. cremoris that cannot be distinguished from Lactococcus lactis subsp. lactis. All these strains were unambiguously discriminated using ON-rep-seq. Two bacterial species: Bacteroides thetaiotaomicron and Lactococcus lactis subsp. cremoris were tested in pairs from different culture collections resulting in all cases in highly reproducible LCp (Supplementary Figure 1). 
Table 01. Results of bacterial isolates identification with ON-rep-seq

\begin{tabular}{|c|c|c|c|}
\hline Bacterium and centrifuge retrieved classification & strain & $\begin{array}{c}\text { average number } \\
\text { of } \\
\text { corrected reads } \\
\text { per sample }\end{array}$ & $\begin{array}{c}\text { average number } \\
\text { of corrected } \\
\text { bases }\end{array}$ \\
\hline Akkermansia muciniphila & DSMZ 22959 & 9 & 4166 \\
\hline Bacillus cereus & 15 & 5 & 8279 \\
\hline Bacillus cereus & NVH 38 & 7 & 7648 \\
\hline Bacillus licheniformis & LMG19409 & 7 & 8603 \\
\hline Bacillus subtilis & In-house strain & 9 & 4707 \\
\hline Bacteriodes cellulosilyticus & DSM 14838 & 9 & 7775 \\
\hline Bacteriodes eggerthii & DSM 20697 & 8 & 5366 \\
\hline Bacteriodes finegoldii & DSM 17565 & 9 & 6720 \\
\hline Bacteriodes intestinalis & DSM 17393 & 13 & 11264 \\
\hline Bacteriodes thetaiotaomicron & ATCC 29148 & 9 & 8597 \\
\hline Bacteroides thetaiotaomicron & DSM $2079^{\top}$ & 12 & 8158 \\
\hline Bacteriodes vulgatus & LMG 17263 & 11 & 8875 \\
\hline Bacteroides fragilis & DSM 2151 & 10 & 6765 \\
\hline Bifidobacterium adolescentis & DSM 20083 & 14 & 7729 \\
\hline Bifidobacterium animalis & DSM 10140 & 16 & 10306 \\
\hline Bifidobacterium bifidum & LMG 11041 & 9 & 9635 \\
\hline Bifidobacterium breve & DSM 20091 & 14 & 10388 \\
\hline Bifidobacterium catenulatum & LMG 11043 & 16 & 13216 \\
\hline Bifidobacterium longum subsp. longum & LMG 13196 & 16 & 7808 \\
\hline Bifidobacterium longum subsp. infantis & DSM 20090 & 17 & 11134 \\
\hline Bifidobacterium pseudocatenulatum & LMG 10505 & 17 & 12818 \\
\hline Escherichia coli & DSM 1058 & 14 & 12897 \\
\hline Lactobacillus acidophilus & LMG $9433^{\top}$ & 5 & 7121 \\
\hline Lactobacillus amylovorus & DSM $20531^{\top}$ & 10 & 10966 \\
\hline Lactobacillus brevis & GGUC30670T & 8 & 12532 \\
\hline Lactobacillus casei & DSM $20011^{\top}$ & 13 & 10238 \\
\hline Lactobacillus fermentum & DSM20052 ${ }^{\top}$ & 9 & 12409 \\
\hline Lactobacillus paracasei subsp. paracasei & NCFB151T & 11 & 13292 \\
\hline Lactobacillus paracasei & In-house strain & 12 & 8728 \\
\hline Lactobacillus plantarum & ATCC $14917^{\top}$ & 7 & 8023 \\
\hline Lactobacillus plantarum & DSM $20174^{\top}$ & 7 & 11459 \\
\hline Lactobacillus rhamnosus & DSM $20021^{\top}$ & 12 & 12199 \\
\hline Lactobacillus sakei subsp. sakei & DSM $20017^{\top}$ & 9 & 3267 \\
\hline Lactococcus lactis subsp. cremoris & MG 1363 & 6 & 7124 \\
\hline Lactococcus lactis subsp. cremoris & Wg2 & 6 & 9094 \\
\hline Leuconostoc mesenteroides subsp. mesenteroides & DSM $20343^{\top}$ & 9 & 9469 \\
\hline Listeria monocytogenes & EGDe & 8 & 6598 \\
\hline Listeria monocytogenes & L028 & 7 & 8209 \\
\hline Listeria monocytogenes & N53-1 & 7 & 5967 \\
\hline Listeria monocytogenes & 12067 & 7 & 8557 \\
\hline Listeria monocytogenes & $42222 / 180$ & 9 & 6378 \\
\hline Pediococcus claussenii & DSM $14800^{\top}$ & 6 & 10290 \\
\hline Pediococcus pentosaceus & DSM $20336^{\top}$ & 8 & 6713 \\
\hline Salmonella enterica subsp. enterica serovar Oranienburg & $0112 \mathrm{~F}$ & 13 & 7316 \\
\hline Salmonella enterica subsp. enterica serovar Typhimurium & U292 & 9 & 12155 \\
\hline Salmonella enterica subsp. enterica serovar Typhimurium & $4 / / 74$ & 9 & 9380 \\
\hline Salmonella enterica subsp. enterica serovar Typhimurium & $\mathrm{C} 5$ & 8 & 5971 \\
\hline Streptoccocus thermophilus & so & 11 & 10764 \\
\hline
\end{tabular}

Subjecting the set of corrected reads for 48 bacterial isolates to centrifuge classifier allowed for unambiguous annotation of all bacteria down to the species and subspecies level.

\section{Paired comparison of LCp can be used for the strain level differentiation}

Five Listeria monocytogenes, four Salmonella enterica (three serovar Typhimurium and one serovar Oranienburg) and two Bacillus cereus strains have been used to evaluate the method for strain level discrimination. We have developed an algorithm (LCpCluster.R) estimating the level of similarity between the pairs of LCp generated by the ON-rep-seq. Among five $L$. monocytogenes strains four unique profiles were identified (Figures 3 and 4). Strains EGDe and LO28 generated identical profiles (Figures 3 A and 4). 

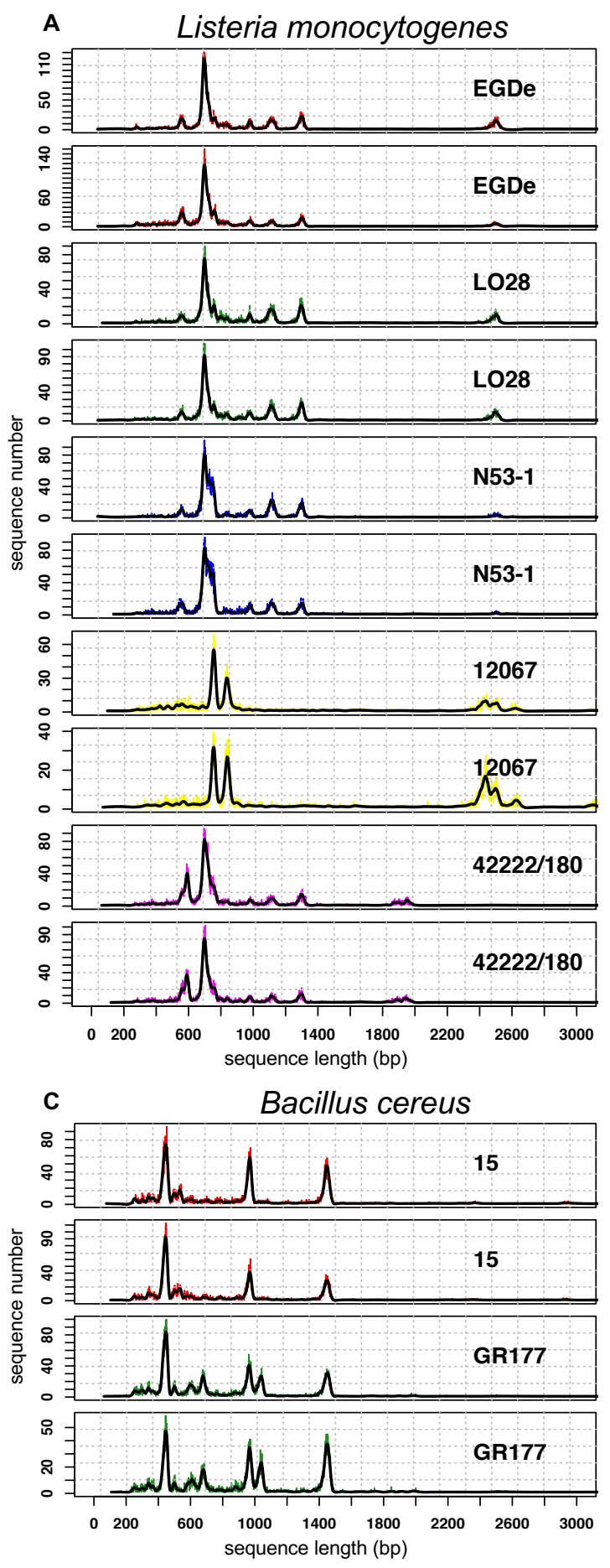
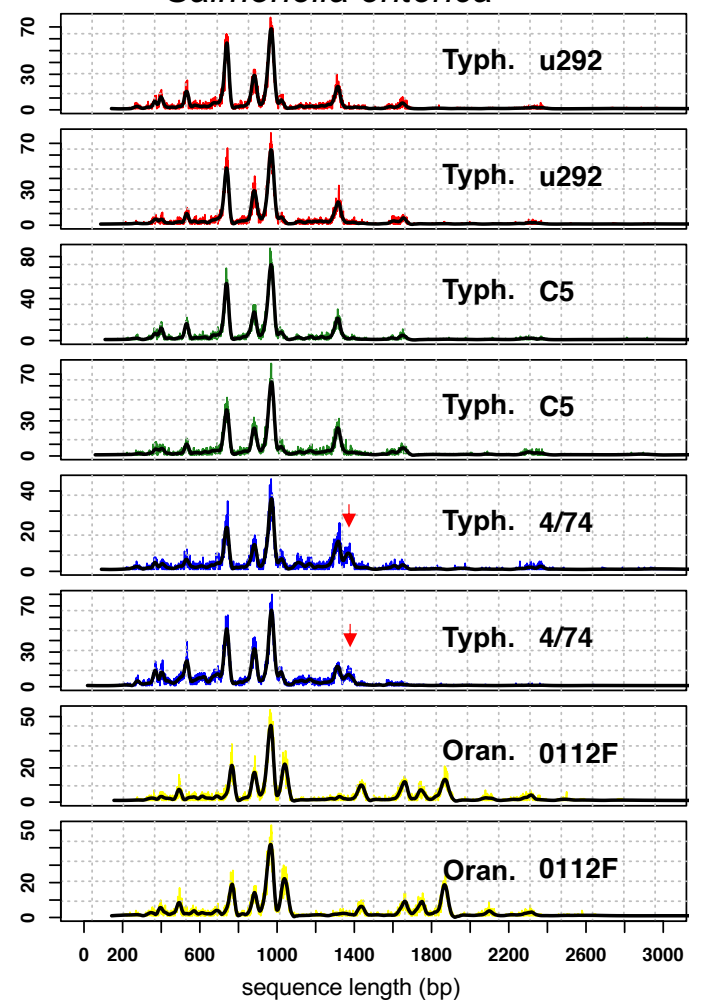

Figure 3. Examples of strain level differentiation using LCp comparison

Oxford Nanopore Technology based rep-PCR amplicon sequencing (ON-rep-seq) of five L. monocytogenes (A), four S. enterica (B) and two B. cereus (C) strains was used to generate read length counts profiles (LCp). All bacterial LCp were produced in duplicates. Consensus sequences from corrected peaks of all 22 samples allowed for unequivocal species and subsbecies level identification. Comparison of LCp revealed four different profiles among the $L$. monocytogenes species. Strains EGDe and LO28 gave highly similar profiles indicating high level of genetical relationship between these two strains (B), what was confirmed by Illumina based shotgun sequencing (orthoANI $=99.9 \%$ ). Similarly C5 and $u 292$ strains of $S$. typhimurium showed the same profiles (orthoANI $=99.9 \%$ ) while two other strains could be classified as different (B). The red arrows indicate additional peak distinguishing the 4/74 strain from u292 and $\mathrm{C} 5$ that was shown to have a prophage origin. The presence of additional peaks in the LCp of GR177 strain allowed for unambiguous differentiation between the two B. cereus strains $(\mathrm{C})$.

No SNPs variants could be detected when comparing consensus sequences of corresponding peaks of all technical replicates (data not shown). Whole genome sequencing (WGS) data have been used to estimate the genetic similarity between EGDe and LO28 strains. The average nucleotide identity (OrthoANI) index between these two genomes reached $99.9 \%$, while $L$. monocytogenes MLST schemes mapped against EGDe and LO28 found only one differing locus (dapE) out of seven tested (Supplementary Table 1). 


\section{Clustering according to K-L divergence-like distance}

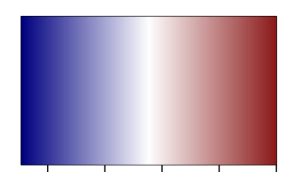

0.2. $0.4 . \quad 0.6 . \quad 0.8 \quad 1.0$ read averaged odds ratio exp(-D_KLsym)

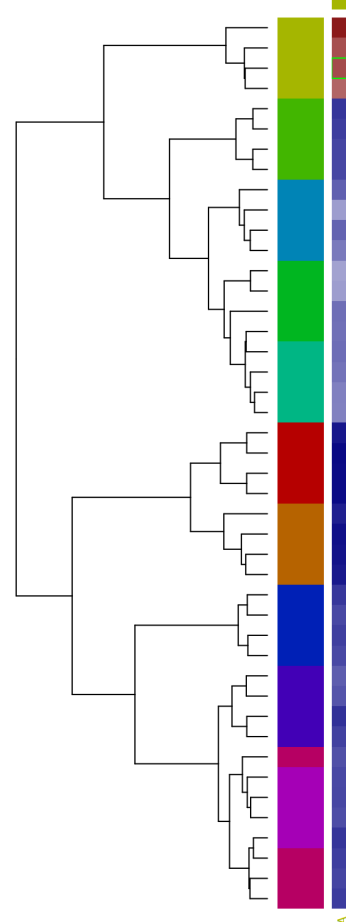

barcode ID
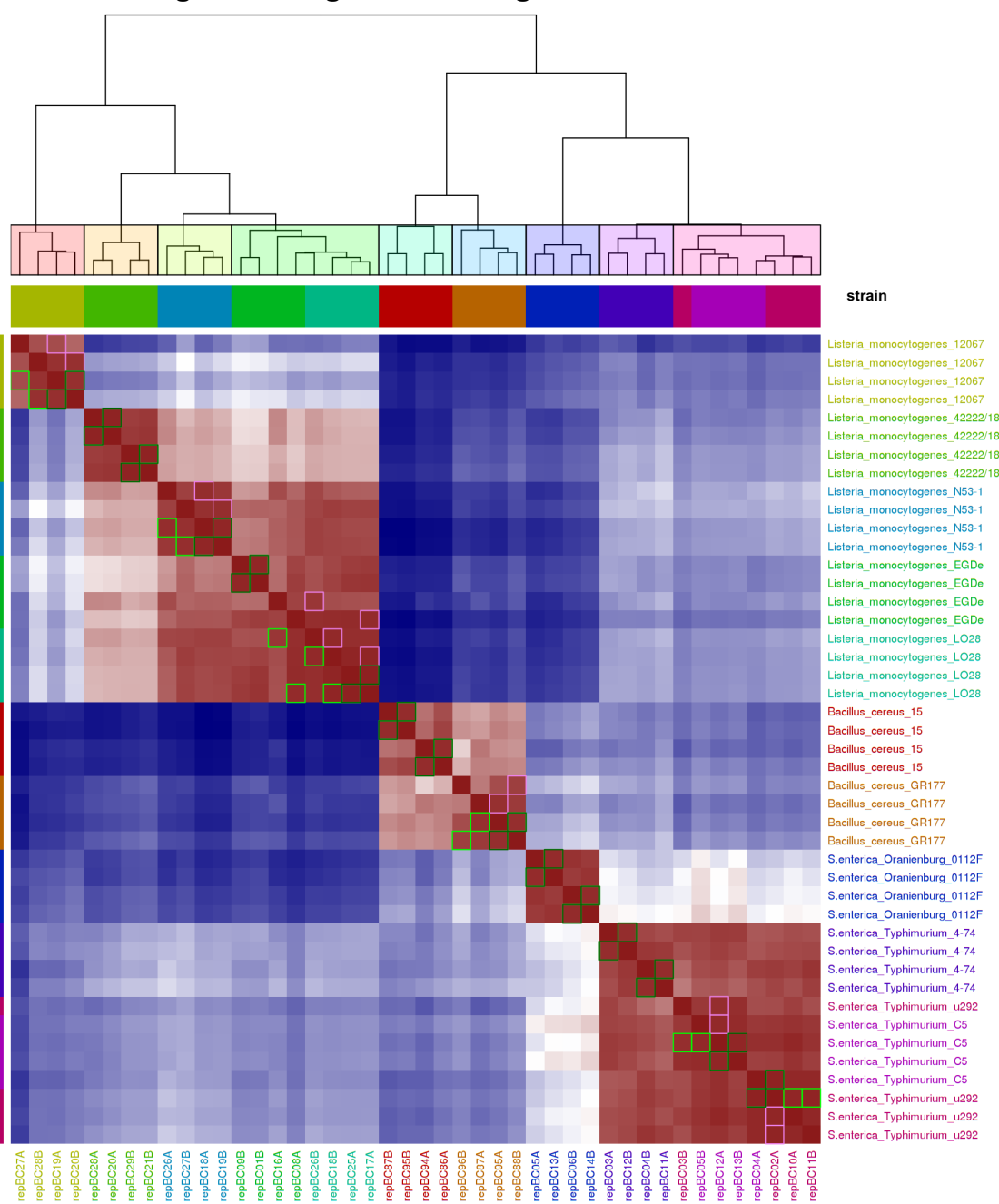

ma smoothing window $=20$, cluster cutoff $=0.09$

Figure 4. Row/Column clustering according to "Ward.D2" hierarchical clustering on D_KLsym distance. Heatmap showing similarity $\left(10^{\wedge}\left(-D_{-}\right.\right.$KLsym $\left.)\right)$, and clusters according to cutoff $=0.09$. Analysis of five Listeria monocytogenes, two Bacillus cereus and four Salmonella enterica serovar Typhimurium strains allowed for species level differentiation in all cases and for strain level differentiation in 8 out of 11 cases. Notably, the presence of the additional peak allowed for unambiguous differentiation of 4/74 from C5 and u292 what was not possible using OrthoANI and MLST analysis based on WGS data. Strain labels colors according to accepted strain similarity derived from visual inspection of profiles in agreement with clustering colors at selected cutoff.

This implies a high level of genetical similarity between the two strains that requires a specific approach to ensure differentiation.

Among four $S$. enterica strains LCpCluster.R recognized three unique profiles (Figure 3 B and 4). Serovar Typhimurium strains u292 and C5 showed the same ON-rep-seq LCp with no SNPs variants in corresponding peaks (data not shown). WGS comparison of these two strains revealed high OrthoANI reaching 99.9\%. Salmonella enterica MLST schemes mapped against genomes of $\mathrm{u} 292$ and C5 also showed the same alleles profiles 
(Supplementary Table 1). This implies that S. enterica strains u292 and C5 could not be straightforwardly distinguished based on their genome using both methods.

Interestingly, serovar Typhimurium strain 4/74 that presented similar LCp to u292 and C5, yet with a clear additional peak in the position $\sim 1370 \mathrm{bp}$ (Figure $3 \mathrm{~B}$ and 4 ) reached OrthoANI above 99.9\% and had the same MLST profile compared to $\mathrm{u} 292$ and C5. In this particular example ON-rep-seq presented higher discrimination power over OrthoANI and MLST analysis based on WGS data. Further investigation of the peak at position 1370bp disclosed that the consensus sequence presented high similarity (blast identity $1372 / 1384 \mathrm{bp} ; 99.1 \%$ ) to SopEФ prophage. Moreover, this sequence could only be found in the draft genome of the 4/74 strain (blast identity $1371 / 1384 \mathrm{bp} ; 99.1 \%$ ) but not in any of the remaining $S$. enterica strains.

Finally, the two $B$. cereus strains generated clearly distinctive LCp and were classified as different strains. (Figure $3 \mathrm{C}$ and 4). The LCp.Cluster results showing grouping according to "Ward.D2" hierarchical clustering on D_KLsym distance of all 48 isolates in four technical replicates from first two runs are given in Supplementary Figure 2.

\section{Benchmarking of the R9.4.1 flow cell indicates the theoretical throughput of ON-rep-seq over 1000 bacterial isolates}

To validate the method, two R9.4.1 flow cells were benchmarked for the maximum possible output generated. The first benchmarked flow cell generated in total over $2.6 \mathrm{M}$ reads (after quality control and demultiplexing). See Supplementary Table 2 for details. In the first four consecutive runs, each lasting $4 \mathrm{~h}$, intertwined with the flow cell washing steps and storage for minimum $24 \mathrm{~h}$ enough data was generated to successfully demultiplex and identify 4x96 bacterial profiles on a single flow cell. The last run generated $0.22 \mathrm{M}$ reads which was enough to detect and correct sequences of 94 out of 96 samples.

The second flow cell generated in total $2.49 \mathrm{M}$ reads respectively $1 \mathrm{M} 0.56 \mathrm{M}$ and $0.87 \mathrm{M}$ for first $(4 \mathrm{~h})$ second $(4 \mathrm{~h})$ and third (12h) run. See Table Supplementary Table 2. All three runs of the second flow cell generated enough data to successfully analyze 96 bacterial profiles. To verify the 
minimum number of reads necessary to analyze all samples the data have been iteratively subsampled and subjected to the analysis with a receiver operating characteristic (ROC) curves to quantify tradeoff between pairwise "same/not-the-same" strain discrimination dependent on clustering cutoff. Throughout the analysis it was noticed that within-strain variance was larger than between-strain variance in cases of small differing features in the latter, and the disproportion of short reads vs long reads in the former case (the observation verified by sample mean read length regression vs sample read count; Supplementary Figure 3). This this disproportion was attributed to the third sequencing run on the reused flow cell, hence the latest repC run was omitted from the cluster analysis and most of ROC curves analysis that follows.

Clustering on different data sets were compared: all, "wo.rep* ${ }^{*}$ (without the third consecutive run: repC), 2\%, 10\%, 20\%, 50\% subsamples. "wo.rep* ${ }^{*}$ " performed best most of the time, though random fluctuations in $50 \%, 20 \%$ and $10 \%$ subsamples overperformed occasionally at single data points. Subsampling to $50 \%$ and 20\% (avg. \#reads/sample 4326 and 1730) performed very similarly to full samples (avg. \#reads/s 8652), while 10\% subsamples performed worse, though still reasonably good, while $2 \%$ subsamples (avg. \#reads/s 173) performed much worse, though relevant information is still present and retrievable even with such a small reads lengths sample (Supplementary Figure $4 \mathrm{C}-\mathrm{H}$ ).

The flow cell benchmarking results showed that $20 \%$ of generated reads (avg. \#reads/sample 1730) were already sufficient to analyze all samples. Notably, the number of isolates that could be analyzed simultaneously on a single flow cell will ultimately depend on the number and position of peaks in LCp (for strain level comparison). Nonetheless, our data demonstrate that the theoretical throughput of the R9.4.1 flow cell ranges between 960 and 1440 isolates depending on the sequencing run performance ( $\sim 1.5 \mathrm{M}$ to $\sim 2.5 \mathrm{M}$ reads respectively). 


\section{Discussion}

The process of fast and accurate bacterial identification, subtyping and strain level differentiation is of high importance in epidemiology, to recognize infection outbreaks, determine its source or follow highly virulent nosocomial pathogens. It is also desired in the food industry to validate quality and safety and to investigate microbiologically complex communities like many fermented foods. For the past three decades the most commonly used and standardized methods became molecular techniques based on DNA analysis 14. Since first described in 1994 Rep-PCR targeting REP and/or repetitive intergenic consensus (ERIC) regions became a widely used methods of DNA typing 12,17 . Its discriminatory power has been shown multiple times to be superior to many other typing methods including ribotyping ${ }^{12,23}$, multilocus enzyme electrophoresis ${ }^{24,25}$, but also biochemical characterization ${ }^{26}$. RepPCR was often shown to have similar or slightly lower discriminatory power than pulse-field gel electrophoresis (PFGE) but was always considered a less laborious and cheaper solution 27,28,29. Among several Rep-PCR options (GTG)5-PCR have reported to be the most robust ${ }^{30}$. Despite welldocumented strain level discrimination power, the main pitfall of Rep-PCR is without a doubt its inability for taxonomic identification, without additional analysis such as $16 \mathrm{~S}$ rRNA gene sequencing, which requires extra laboratory work and significantly increases time and cost of the analysis. Moreover, such strategy relays entirely in the discriminatory power of 16S rRNA gene that does not always allow for species level identification.

The massive leap in DNA sequencing methods made within the last decade heralded the inevitable decline of many "old-fashioned" DNA finger-print based typing methods. A single HiSeq $X$ instrument (Illumina) has a capacity to sequence about 35,000 average size bacterial genomes with 100 times coverage in a single run (Illumina.com). Yet, notwithstanding the immense potential, this technology is still not meant for fast, routine and cost-effective typing of bacteria. It is mainly due to the high equipment cost, low flexibility requiring collection of multiple samples (from dozens to thousands depending on the platform), relatively long runtime and complex data analysis. The 
portable, USB powered MinION offered by Oxford Nanopore Technologies is so far the cheapest $(\sim 1000 \$)$ sequencing platform on the market. Its main advantage besides the price is the possibility to generate ultra-long reads with the longest ones crossing $1 \mathrm{Mb}$. Nonetheless, there are two main reasons why ONT have not yet become the first choice of a sequencing strategy in many laboratories. First is the relatively high base calling error rate of a single DNA molecule and second, a relatively low throughput compared to many other platforms ${ }^{10}$. Our method have largely solved these two hindrances, allowing ONT to be exploited for accurate, large scale and detailed identification of bacteria. Highly reproducible amplification of regions flanked by REP elements not only solved the main problem with sequencing redundancy one needs to deal during WGS, but also enabled single base error correction owing it to its random nature. ON-rep-seq offers the well documented discrimination power of the DNA fingerprint analysis, but also for the first time full access to the "hidden information" within each band DNA sequence in quality crossing 99\% accuracy. Since each isolate composes on average of 10 corrected consensus reads with an average length of $1 \mathrm{~Kb}$ this information can be used for highly accurate taxonomic identification. Even if one of the reads would not find a hit in a database, there are still several others to ensure classification. As shown here, all 48 isolates have been accurately assigned to the species and sub-species level. Also, for the first time users will be able to easily determine contaminations in case one or several peaks would turn out to belong to another organism. Lastly, by reducing the number of samples and increasing the coverage one could achieve even higher accuracy of the consensus sequence what could be used to assess presence of SNPs in profiles of closely related strains. This might be an additional source of previously unknown discrimination repository of Rep-PCR in some unique cases.

We have demonstrated here that ON-rep-seq successfully identified and differentiated between Listeria monocytogenes and Salmonella enterica serovar Typhimurium strains. Both species are of special epidemiological importance and model organisms for host-pathogen infection 31 32. Rep-PCR was previously recommended method for subtyping of $L$. monocytogenes and 
S. enterica with a similar discrimination power of PFGE or RAPD ${ }^{29,33}$. The only two undistinguished pairs of strains were L. monocytogenes EGDe from LO28 and Salmonella enterica C5 from u292. Paired comparison of their genomes revealed high level of similarity (OrthoANI > 99.9\%) further confirmed with the MLST indicating that genetic diversity between these two strains could be allocated in SNPs. Regrettably, none of these SNPs were found by comparing sequence within the peaks. Although ON-rep-seq cannot discriminate between strains that differs solely with SNPs it can be used for fast and cost-effective screening of multiple isolates to select those of identical profiles that should be subjected for deep sequencing saving resources, money and time.

Interestingly ON-rep-seq was shown to be superior to traditional WGS analysis in distinguishing between S. enterica u292 and 4/74. The OrthoANI between these two strains reached $>99.9 \%$ with identical MLST profiles. This makes it very challenging to differentiate between $u 292$ and 4/74 at the strain level using WGS 34,35 . However, comparison of ON-rep-seq based LCp allowed for clear and unambiguous differentiation between the two strains. The peak allowing this distinction was shown to be a mobile element with high similarity to a prophage. It was previously demonstrated that large fractions of genetic variation in in Salmonella strains is allocated in variable genomic regions and islands that encompass phage insertions ${ }^{36}$.

Presented in this work barcodes enable accurate tagmentation of 96 isolates, but our data demonstrate that even about 1000 barcodes could be used on a single R9 flow cell. We have benchmarked two R9.4.1 flow cells to estimate the maximum possible output and cost per isolate. Since the flow cell price ranges between $475 \$-900 \$$ (depending on the bundle offer) the sole cost of sequencing assuming highest output would range between $0.40-0.75 \$$. It is important to mention that the maximum data output will vary depending on the flow cell viability that may be affected by multiple washing steps. Furthermore, it was demonstrated herein that consecutive usage of the flow cell may be a source of an increased run effect. Therefore, the best performance of ON-repseq could be achieved if for example 96x10 barcodes were used in a single 
run lasting for maximum time (48h). It seems however that the new gadget offered by ONT called Flongle, promised to be released this year, could be the most optimal and user-friendly solution for ON-rep-seq (nanoporetech.com). Flongle is an adapter for MinION with one-quarter throughput of a R9 flow cell but price not crossing $100 \$$. This means that up to $3 \times 96$ isolates could be analyzed in a single run for about $0.35 \$$ per sample. Naturally, the user could then choose to sequence less isolates but ensure even better coverage.

In summary, we present here the DNA enrichment and barcoding method called ON-rep-seq (from: Oxford Nanopore based Rep-PCR based sequencing) that in combination with ONT sequencing platforms allows for highly cost effective, bulk screening of bacterial isolates with species and strain level resolution. We believe that ON-rep-seq has a potential to become a modern standard molecular based method with multiple applications in research, industry and medicine. By sharing it to other users we are looking forward for thorough validation of many more bacterial species, optimization of sequencing protocols and pipelines. We hope that conjoined effort of multiple users will also allow for the development of ON-rep-seq consensus reads database facilitating in the even faster and simplified identification. 


\section{Methods}

\section{Wet laboratory}

\section{Rep-PCR-1}

Bacterial genomic DNA was extracted using GenElute ${ }^{\mathrm{TM}}$ Bacterial Genomic Kit (Sigma Life Science, Darmstadt, Germany) according to the manufacturer's instructions. In total 48 isolates represented by 38 different bacterial species were subjected for the analysis in duplicates in each of the three runs giving six technical replicates per isolate. The barcodes order was shifted during preparation of each library to ensure that every technical replicate is tagged with different barcode sequence. Three strains of Salmonella enterica, five Listeria monocytogenes and two Bacillus cereus strains have been used to evaluate the ability of the method for strain level differentiation. The detailed list of bacteria used for the analysis is given in Table1 while ON-rep-seq LCp in Supplementary Figure 1. The Rep-PCR reaction mix contained $5 \mu \mathrm{l}$ PCRBIO HiFi buffer (5x), $0.25 \mu$ of PCRBIO HiFi Polymerase (PCR Biosystems Ltd, London, United Kingdom), $4 \mu$ of (GTG)5 primers $(5 \mu \mathrm{M}), 1 \mu \mathrm{l}$ of DNA $(\sim 20 \mathrm{ng} / \mu \mathrm{l})$ and nuclease-free water to a total volume of $25 \mu \mathrm{l}$. The Rep-PCR thermal conditions were optimized as follows: Denaturation at $95^{\circ} \mathrm{C}$ for $5 \mathrm{~min} ; 30$ cycles of $95^{\circ} \mathrm{C}$ for $30 \mathrm{~s}, 45^{\circ} \mathrm{C}$ for $1 \mathrm{~min}$ and $62^{\circ} \mathrm{C}$ for $4 \mathrm{~min}$; followed by final elongation at $72^{\circ} \mathrm{C}$ for $5 \mathrm{~min}$. It is important to note that several polymerases have been tested in order to shorten the elongation time without compromising the longest amplicons. With current settings, the PCR takes less than 3h on SureCycler 8800 (Agilent, CA, USA).

\section{Barcoding by dual-stage Rep-PCR-2}

We have designed 96 ONT compatible barcodes (Supplementary Table 3) with 10bp spacer separating ONT motor protein adapter from the barcode sequence and (GTG)5 pairing region. The spacer was added to ensure higher tolerance for the low-quality at the beginning of the sequence entering the pore and thus higher recovery of barcode sequence. At the same time the spacer sequence was designed to prevent creations of stem-loops in relatively long primers during low temperature annealing step. The Rep-PCR reaction mix contained $12 \mu \mathrm{l}$ of PCRBIO UltraMix (PCR Biosystems Ltd, 
London, United Kingdom) $2 \mu$ of corresponding repBC primer $(10 \mu \mathrm{M}), 1 \mu \mathrm{l}$ of PCR product from Rep-PCR-1 and nuclease-free water to a total volume of 25 $\mu \mathrm{l}$. Incorporation of ONT compatible adapters (Supplementary Table 3) was performed using dual-stage PCR where first 3 cycles provide optimal annealing of (GTG)5 regions, while next 10 cycles allow for best hybridization of full adapters in consecutive cycles: Denaturation $5 \mathrm{~min} ; 3$ cycles of $95^{\circ} \mathrm{C}$ for $30 \mathrm{~s}, 45^{\circ} \mathrm{C}$ for $1 \mathrm{~min}$ and $62^{\circ} \mathrm{C}$ for $4 \mathrm{~min}$; followed by 10 cycles of $95^{\circ} \mathrm{C}$ for 30 $\mathrm{s}, 65^{\circ} \mathrm{C}$ for $1 \mathrm{~min}$ and $72^{\circ} \mathrm{C}$ for $4 \mathrm{~min}$ and final elongation at $72^{\circ} \mathrm{C}$ for $5 \mathrm{~min}$.

\section{Library preparation and ONT-sequencing}

After Rep-PCR-2 samples were pooled using $10 \mu \mathrm{l}$ of each sample. Note that samples were not pooled in equimolar concentration due to expected differences in length of amplified regions between the samples. However, it is

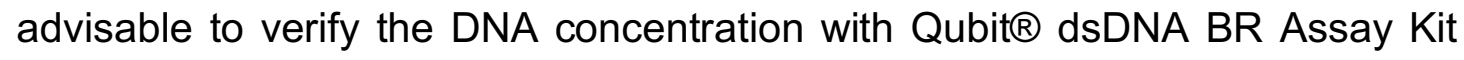
(Life Technologies, CA, USA) for the quality control of the Rep-PCR-2 step. The measurement was performed with Varioskan Flash Multimode Reader (Thermo Fischer Scientific, MA, USA). Fluorescence was measured at $485 / 530 \mathrm{~nm}$.

The pooled library was cleaned with AMPure XP beads (Beckman Coulter Genomic, CA, USA) in volumes 100:50 $\mu$ respectively. The bead pellet was washed with $80 \%$ ethanol and re-suspended in $100 \mu$ l of nuclease-free water. The bead washing step was added to shift the proportion of short to long reads that is multi-template PCR specific feature and to remove primerdimers. The pooled and bead-purified library was measured with Qubit $\circledast$ dsDNA HS Assay Kit (Life Technologies, CA, USA) and 66 ng of library was used as an input to the End-prep step in 1D amplicon by ligation protocol (ADE_9003_v108_revT_180ct2016) with one adjustment: 80\% ethanol instead of $70 \%$ was used for all washing steps.

To validate our method, we have benchmarked two R9.4.1 flow cells for the maximum possible output generated. First flow cell was used 10 days after the delivery in five consecutive runs, each lasting $4 \mathrm{~h}$, intertwined with the flow cell washing steps and storage for minimum 24h (QC 1347 active pores). Second flow cell was used 44 days after the delivery in three consecutive runs lasting respectively $4 \mathrm{~h}, 4 \mathrm{~h}$ and $12 \mathrm{~h}$ (to collect maximum amount of data from 
declining flow cell; QC 1105 active pores). After each run the flow cell was washed according to the manufacturer's instructions and a new library was prepared and loaded. In order to evaluate the possibility of barcode specific amplification during Rep-PCR-2 step, all samples received different barcode in consecutive sequencing runs. The data from the first benchmarked flow cell were used solely to test the optimal concentration of DNA needed and viability of the flow cell while data from the second flow cell are presented herein and can be downloaded from SRA NCBI repository (\#SUB4333515). 


\section{Data analysis}

\section{Data collection, base calling, demultiplexing and trimming}

Data were collected using MinKnow 1.10.23. The amount of data collected in both R.9.4 flow cells is listed in Supplementary Table 2. Albacore v2.1.3 pipeline was used to base call raw fast5 to fastq. Porechop v0.2.2 was used for adapters trimming and samples demultiplexing. Porechop settings together with the list of custom adapters (adapters.py) compatible with oligos given in Supplementary Table 3 are available at (bitbucket.org/modelscat/on-rep-seq) The script allows for demultiplexing up to 96 barcodes and trimming of: ONT adapters, custom spacers and tandem repeats of (GTG)n.

\section{Correction and base location of peaks}

Peaks are identified in LCp expressed as sequencing length ( $x$-axis) by number of reads (y-axis) by fitting local third order polynomials in a sliding window of size $1 / 50$ of the $x$-span across the $x$-axis, followed by calculation of the first- and second order derivatives. The position of a peak is identified at the $\mathrm{x}$-axis where the first derivative is zero and the second derivative is negative. Only peaks with intensity higher than baseline, defined as a moving boxcar (zero order polynomial) in a broad window (4 times the size of the window used for calculation of the derivative) are used for further analysis. The identified peaks are ordered based on the height, and a representative fragment are used for data base matching.

\section{Reads correction within a peak}

Sequences containing quality scores (fastq files) resolved within each peak were retrieved using Cutadapt v1.15 ${ }^{37}$, and corrected with Canu v1.6 ${ }^{22}$ using the following parameters: genomeSize $=5 \mathrm{k}$, minimumReadLength $=200$, correctedErrorRate $=0.05$, corOutCoverage $=5000$, corMinCoverage $=2$ and minOverlapLength $=50$. The corrected reads were sorted-by-length and clustered with UCLUST (cluster_fast) from USEARCH v10.0 ${ }^{38}$, using the following options: -id of 0.9, -minsl of 0.8, -sizeout, and min_cons_pct of 20. Subsequently, consensus sequences were sorted-by-size (coverage) and 
those with a minimum coverage-size of $50 \times$ were kept for downstream analyses.

\section{Classification}

Centrifuge 1.0.3 microbial classification engine was used for labelling of corrected reads.

\section{Comparison of LCp}

The identification of a good distance measure on read length counts profiles (LCp) was approached by considering them as approximating samples of their underlying sampling distributions. Ideally one would like to understand processes involved in signal peaks and noise formation, thus a priori distributions could be postulated, and later optimized for profiles posteriors. Primarily, empirical discrete length distributions were smoothed with window moving average (ma). Selection of ma window size was done by computing the average jitter of all profiles: an average number of times when profile's discrete derivative changes sign (change to 0 was counted as 0.5 ). From mean jitter plot "ma" window size was selected to 20 , the point of the lowest second derivative, after which second derivative stabilized closely around 0 , meaning the information (jitter) loss due to increasing of window size became relatively low and more constant (Supplementary Figure 4 A, B). Next with each LCp assigned was a "ma" smoothed and probability-normalized "distribution profile" Dp.

Stability problems around $\#$ reads $(i)=D p(i)=0$ are avoided by considering a mixture of "ma" smoothed Dp and the uniform read lengths distribution (in a considered range 150 to $3000 \mathrm{bp})$ with proportions $(0.99,0.01)$. The distance between two samples, reads lengths-based, was defined as a function of LCp_1, Dp_1, LCp_2, Dp_2. One natural approach was to consider the probability of sampling LCp_1 from Dp_2, however for the distance to be comparable between samples of different read counts it needed to be normalized by total read count. Resulting is the following logarithmic formula: 


$$
D_{\mathrm{nprob}}\left(L C p_{1} \| D p_{2}\right)=-\sum_{i} \frac{L C p_{1}(i)}{\# L C p_{1}} \log _{10} D p_{2}(i)=-\sum_{i} P_{L C p_{1}(i)} \log _{10} D p_{2}(i),
$$

The above formula is however not centralized because the distance of a sample to itself is not 0 but it is rather equal to sample's smoothed entropy. Centralization of this distance yields distance very similar to Kullback-Leiber divergence of probabilities, which is proposed for the distance between LCp, as follows:

$$
D_{\mathrm{KL}}\left(L C p_{1} \| D p_{2}\right)=-\sum_{i} P_{L C p_{1}(i)} \log _{10} \frac{D p_{2}(i)}{D p_{1}(i)}
$$

In the following clustering analysis, we use the symmetrized version:

$$
D_{\mathrm{KLsym}}\left(L C p_{1}, L C p_{2}\right)=\frac{D_{\mathrm{KL}}\left(L C p_{1} \| D p_{2}\right)+D_{\mathrm{KL}}\left(L C p_{2} \| D p_{1}\right)}{2}
$$

\section{Analysis of D_KLsym distance between peaks profiles performance on bacterial LCp generated with ON-rep-seq}

Validation of $\mathrm{KL}$ based distance on $\mathrm{LCp}$ by hierarchical clustering was performed on sequencing results where clusters were compared with downto-strain sample labels. To promote clusters with low variance around centroids "Ward.D2" clustering method was selected and performed with modified "heatmap3" R library. Figure 4 shows clusters recovered with cutoff $=0.09$ where all $L$. monocytogenes, B. cereus and S. enterica strains with clearly visible feature peaks were properly clustered.

\section{Whole genome sequencing data analysis}

Complete or draft genomes L. monocytogenes EGDe (\#NC_003210.1) and LO28 (\#AARY02000001.1-2001127.1); S. enterica serovar Typhimurium ST4/74 (\#CP002487.1) and u292 (\#ERR277220) were downloaded from public databases and compared using OrthoANI ${ }^{8}$.

Salmonella enterica MLST schemes (internal fragments and their alleles) hosted at PubMLST.org were mapped against genomes of U292 
(\#ERR277220) and 4/74 ((\#CP002487.1), as well as the assemble contigs of C5 strain included in this study.

For strain C5, DNA was subjected to library preparation (Nextera XT kit, following manufacturer procedures) and sequencing on Illumina NextSeq platform. High-quality reads ( $>95 \%$ quality and minimum size of 50 nt using Trimmomatic v0.35 ${ }^{39}$ were de-convoluted from phiX174 controls reads (-id: 0.97, -query_cov: 0.97) and dereplicated using USEARCH v10 40. Subsequently, reads were assembled into contigs using Spades v3.5.0 ${ }^{41}$. Contigs with a minimum size of 10,000 bp generated for C5 strain, and in addition to the publicly available U292 and 4/74 putative genomes, were subjected to MLST analysis on the CLC Genomics Workbench v11.1 using a minimum alignment length of $400 \mathrm{bp}$ and high level of alignment stringency. 


\section{Acknowledgements}

To Pernille Johansen for inspirational conversation. To Witold Kot and Aarhus University for generating WGS data. To Basheer Yousef Aideh for providing access to the broad bacterial collection. To Henrik Siegumfeldt for help in editing.

\section{Authors contributions}

L.K conceived the presented idea, participated in the data analysis, developed the wet-lab protocol, participated in pipeline development, designed figures, wrote the manuscript. J.L.C.M participated in pipeline development, data analysis and interpretation, tables generation, contributed in manuscript writing. D.N.M participated in optimization of the wet-lab protocol, data analysis and interpretation. M.B.M participated in optimization of the wet-lab protocol. M.A.R participated in the data analysis and interpretation, pipeline development, contributed in manuscript writing. M.S participated in the data analysis, interpretation, pipeline development, designed several figures, contributed in manuscript writing. D.S.N supervised the work, participated in formulation of the initial idea, contributed in manuscript writing. All authors edited and approved the final version of the manuscript.

\section{Corresponding authors}

Lukasz Krych or Josué L. Castro-Mejia or Dennis S. Nielsen

\section{Software and code}

ON-rep-seq pipeline is available at: bitbucket.org/modelscat/on-rep-seq

\section{Data:}

Fastq files can be downloaded from SRA NCBI repository (\#SUB4333515) 


\section{References}

1. Marx, V. Microbiology: The road to strain-level identification. Nat. Methods 13, 401-404 (2016).

2. Janda, J. M. \& Abbott, S. L. 16S rRNA gene sequencing for bacterial identification in the diagnostic laboratory: Pluses, perils, and pitfalls. Journal of Clinical Microbiology 45, 2761-2764 (2007).

3. Hrabák, J., Chudác ková, E. \& Walková, R. Matrix-assisted laser desorption ionization-time of flight (MALDITOF) mass spectrometry for detection of antibiotic resistance mechanisms: From research to routine diagnosis. Clin. Microbiol. Rev. 26, 103-114 (2013).

4. Rodriguez, M. et al. Discriminatory Indices of Typing Methods for Epidemiologic Analysis of Contemporary Staphylococcus aureus Strains. Med. 94, e1534 (2015).

5. Sandrin, T. R., Goldstein, J. E. \& Schumaker, S. MALDI TOF MS profiling of bacteria at the strain level: A review. Mass Spectrometry Reviews 32, 188-217 (2013).

6. Miller, J. R. et al. Hybrid assembly with long and short reads improves discovery of gene family expansions. BMC Genomics 18, (2017).

7. Carlisle, E. M. et al. Murine Gut Microbiota and Transcriptome Are Diet Dependent. Ann. Surg. 257, 1 (2012).

8. Lee, I., Kim, Y. O., Park, S. C. \& Chun, J. OrthoANI: An improved algorithm and software for calculating average nucleotide identity. Int. J. Syst. Evol. Microbiol. 66, 1100-1103 (2016).

9. Scholz, M. et al. Strain-level microbial epidemiology and population genomics from shotgun metagenomics. Nat. Methods 13, 435-438 (2016).

10. Laver, T. et al. Assessing the performance of the Oxford Nanopore Technologies MinION. Biomol. Detect. Quantif. 3, 1-8 (2015).

11. Stern, M. J., Ames, G. F. L., Smith, N. H., Clare Robinson, E. \& Higgins, C. F. Repetitive extragenic palindromic sequences: A major component of the bacterial genome. Cell 37, 1015-1026 (1984).

12. Versalovic, J., Schneider, M. \& Bruijn, F. De. Genomic fingerprinting of bacteria using repetitive sequence-based polymerase chain reaction. Methods Mol. Cell. Biol. 25-40 (1994).

13. Versalovic, J., Woods, C. R., Georghiou, P. R., Hamill, R. J. \& Lupski, J. R. DNA-based identification and epidemiologic typing of bacterial pathogens. Archives of Pathology and Laboratory Medicine 117, 10881098 (1993).

14. Olive, D. M. \& Bean, P. Principles and applications of ligation mediated PCR methods for DNA-based typing of microbial organisms. J. Clin. Microbiol. 37, 1661-1669 (1999).

15. De Vuyst, L. et al. Validation of the (GTG)5-rep-PCR fingerprinting technique for rapid classification and identification of acetic acid bacteria, with a focus on isolates from Ghanaian fermented cocoa beans. Int. J. Food Microbiol. 125, 79-90 (2008).

16. Gevers, D., Huys, G. \& Swings, J. Applicability of rep-PCR fingerprinting for identification of Lactobacillus species. FEMS Microbiol. Lett. 205, 31-36 (2001).

17. Ishii, S. \& Sadowsky, M. J. Applications of the rep-PCR DNA fingerprinting technique to study microbial diversity, ecology and 
evolution. Environ. Microbiol. 11, 733-740 (2009).

18. Tafvizi, F. \& Tajabadi Ebrahimi, M. Application of repetitive extragenic palindromic elements based on PCR in detection of genetic relationship of lactic acid bacteria species isolated from traditional fermented food products. J. Agric. Sci. Technol. 17, 87-98 (2015).

19. Wise, M. G. et al. Predicting Salmonella enterica serotypes by repetitive sequence-based PCR. J. Microbiol. Methods 76, 18-24 (2009).

20. Nurhayati, Priyambada, I. D., Radjasa, O. K. \& Widada, J. Repetitive element palindromic PCR (Rep-PCR) as a genetic tool to study diversity in amylolytic bacteria. Adv. Sci. Lett. 23, 6458-6461 (2017).

21. Healy, M. et al. Microbial DNA typing by automated repetitivesequence-based PCR. J. Clin. Microbiol. 43, 199-207 (2005).

22. Koren, S. et al. Canu: Scalable and accurate long-read assembly via adaptive K-mer weighting and repeat separation. Genome Res. 27, 722-736 (2017).

23. Appuhamy, S., Parton, R., Coote, J. G. \& Gibbs, H. A. Genomic fingerprinting of Haemophilus somnus by a combination of PCR methods. J. Clin. Microbiol. 35, 288-291 (1997).

24. Woods, C. R., Versalovic, J., Koeuth, T. \& Lupski, J. R. Analysis of relationships among isolates of Citrobacter diversus by using DNA fingerprints generated by repetitive sequence-based primers in the polymerase chain reaction. J. Clin. Microbiol. 30, 2921-2929 (1992).

25. Harvey, J., Norwood, D. E. \& Gilmour, A. Comparison of repetitive element sequence-based PCR with multilocus enzyme electrophoresis and pulsed field gel electrophoresis for typing Listeria monocytogenes food isolates. Food Microbiol. 21, 305-312 (2004).

26. Clarridge, J. E. et al. Strategy to detect and identify Bartonella species in routine clinical laboratory yields Bartonella henselae from human immunodeficiency virus- positive patient and unique Bartonella strain from His cat. J. Clin. Microbiol. 33, 2107-2113 (1995).

27. Gunawardana, G. A., Townsend, K. M. \& Frost, A. J. Molecular characterisation of avian Pasteurella multocida isolates from Australia and Vietnam by REP-PCR and PFGE. Vet. Microbiol. 72, 97-109 (2000).

28. Northey, G., Gal, M., Rahmati, A. \& Brazier, J. S. Subtyping of Clostridium difficile PCR ribotype 001 by REP-PCR and PFGE. J. Med. Microbiol. 54, 543-547 (2005).

29. Chou, C. H. \& Wang, C. Genetic relatedness between Listeria monocytogenes isolates from seafood and humans using PFGE and REP-PCR. Int. J. Food Microbiol. 110, 135-148 (2006).

30. Mohapatra, B. R. \& Mazumder, A. Comparative efficacy of five different rep-PCR methods to discriminate Escherichia coli populations in aquatic environments. Water Sci. Technol. 58, 537-547 (2008).

31. Hamon, M., Bierne, H. \& Cossart, P. Listeria monocytogenes: A multifaceted model. Nature Reviews Microbiology 4, 423-434 (2006).

32. Leekitcharoenphon, P., Nielsen, E. M., Kaas, R. S., Lund, O. \& Aarestrup, F. M. Evaluation of whole genome sequencing for outbreak detection of salmonella enterica. PLoS One 9, (2014).

33. Albufera, U., Bhugaloo-Vial, P., Issack, M. I. \& Jaufeerally-Fakim, Y. Molecular characterization of Salmonella isolates by REP-PCR and 
RAPD analysis. Infect. Genet. Evol. 9, 322-327 (2009).

34. Konstantinidis, K. T. \& Tiedje, J. M. Genomic insights that advance the species definition for prokaryotes. Proc. Natl. Acad. Sci. 102, 25672572 (2005).

35. Konstantinidis, K. T., Ramette, A. \& Tiedje, J. M. Toward a more robust assessment of intraspecies diversity, using fewer genetic markers. Appl. Environ. Microbiol. 72, 7286-7293 (2006).

36. Jacobsen, A., Hendriksen, R. S., Aaresturp, F. M., Ussery, D. W. \& Friis, C. The Salmonella enterica Pan-genome. Microbial Ecology 62, 487-504 (2011).

37. Martin, M. Cutadapt removes adapter sequences from high-throughput sequencing reads. EMBnet.journal 17, 10 (2011).

38. Edgar, R. C. Search and clustering orders of magnitude faster than BLAST. Bioinformatics 26, 2460-2461 (2010).

39. Bolger, A. M., Lohse, M. \& Usadel, B. Trimmomatic: A flexible trimmer for Illumina sequence data. Bioinformatics 30, 2114-2120 (2014).

40. Edgar, R. C. Sequence analysis Search and clustering orders of magnitude faster than BLAST. 26, 2460-2461 (2010).

41. Bankevich, A. et al. SPAdes: A New Genome Assembly Algorithm and Its Applications to Single-Cell Sequencing. J. Comput. Biol. 19, 455477 (2012). 


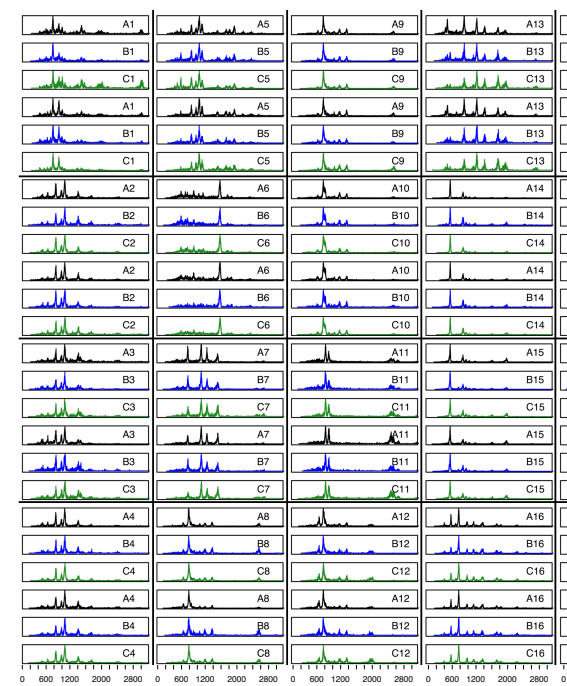

\section{replicate bacterium}

A1 B1 C1 Streptoccocus thermophilus

A2 B2 C2 Salmonella enterica subsp. enterica serovar Typhimurium

A3 $\mathrm{B} 3 \mathrm{C} 3$

A4 B4 C4

A5 $\mathrm{B} 5 \mathrm{C} 5$

$\mathrm{A} 6 \mathrm{~B} 6 \mathrm{C6}$

A7 $\mathrm{B} 7 \mathrm{C7}$

A8 $\mathrm{B} 8 \mathrm{C} 8$

A9 B9 C9

Salmonella enterica subsp. enterica serovar Typhimurium

Salmonella enterica subsp. enterica serovar Typhimurium

Salmonella enterica subsp. enterica serovar Oranienburg

Pediococcus pentosaceus

Pediococcus claussen

Listeria monocytogenes

Listeria monocytogene

A11 B11 C11 Listeria monocytogenes

A12 B12 C12 Listeria monocytogenes

A14 14 C13 Leuconostoc mesenteroides mesenteroides

A14 B14 C14 Laclococcus lactis subsp. cremonis

A15 B15 C15 Lactococcus lactis subsp. cremonis

A16 B16 C16 Lactobacillus sakei subsp. sak

A17 B17 C17 Lactobacillus rhamnosus

A18 B18 C18 Lactobacillus plantarum

A19 B19 C19 Lactobacilus plantanm

ei subsp. paracase

A21 B21 C21 Lactobacillus fermentum

A22 B22 C22 Lactobacillus paracasei

A23 B23 C23 Lactobacillus casel

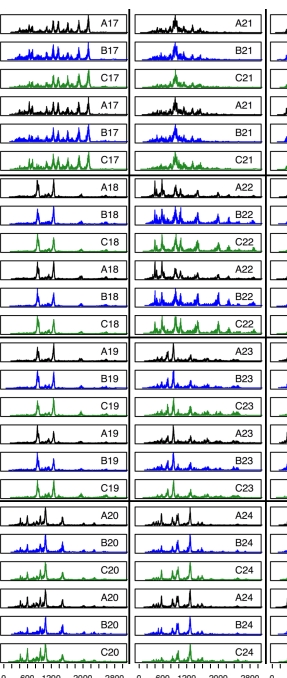

strain

so

u292

$4 / / 74$
C5

$0112 \mathrm{~F}$

DSM20336T

DSM14
EGDe
L028

N53-1

12067

DS222/180

MG1363

Wg2

DSM20017T

ATCC14917T, LLFH15

NCFB151T, LLFH13

DSM20052

Own

DSM20011T, LLFH4

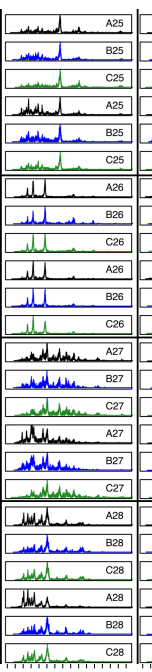

replicate

A25 B25 C25

A26 B26 C26

A27 B27 C27

A28 B28 C28

A29 B29 C29

A30 B30 C30

A31 B31 C31

A 32 B 32 C 32

А 33 B 33 C 33

A 34 B 34 C 34

A 35 B 35 C 35

A 36 B 36 C 36

А $37 \mathrm{~B} 37 \mathrm{C} 37$

A38 B38 C38

A

A40 B40 C40

A41 B41 C41

A 42 B 42 C 42

A44 B44 C44

A44 B44 C44

A45 B45 C45

A47 B 47 C 47

A47 B47 C47
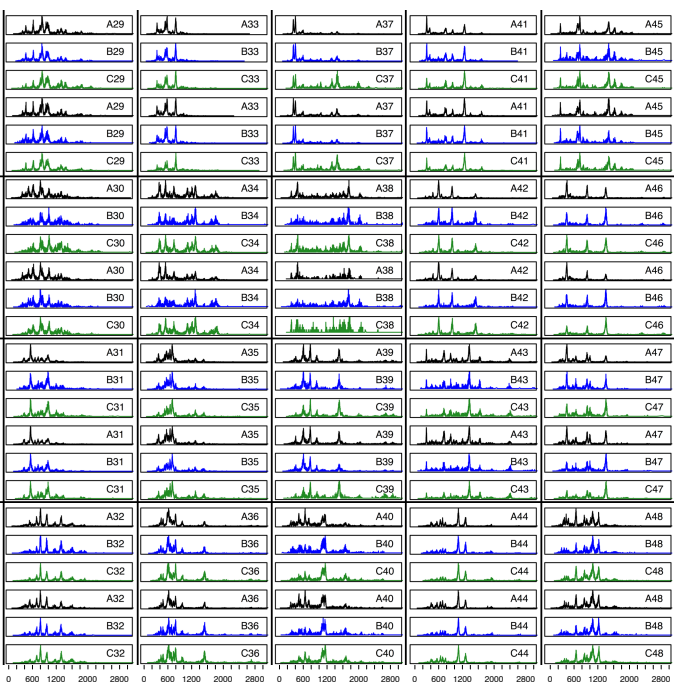

bacterium

Lactobacillus amylovorus

Lactobacillus acidophilus

Escherichia coli

Bifidobacterium pseudocatenulatum

Bifidobacterium longum

Bifidobacterium catenulatum

Bifidobacterium breve

Bifidobacterium bifidum

Bifidobacterium animalis
Bifidobacterium adolescentis

Bacteroides thetaiotaomicron

Bacteroides fragilis

Bacteriodes vulgatus
Bacteriodes thethaiotaomicron

Bacteriodes intestinalis

Bacteriodes finegold

Bacteriodes eggerthii

Bacillus subtilis

Bacillus lichniformis

Bacillus cereus

Bacillus cereus
Akkermansia muciniphila train

DSM20531T

LMG 9433T, T30

DSM1058

LMG10505

DSM20090

MG13196

LMG11043

DSM20091

LMG11041

DSM10140

DSM20083

DSM2079

DSM2151

LMG17263

DSM2079

DSM17393

DSM17565

DSM20697

(DST1483

CMG19409

15

38 (GR177)

DSMZ 22959

Supplementary Figure 1. LCp generated for 48 bacterial isolates using Oxford Nanopore Technology based rep-PCR amplicon sequencing (ON-rep-seq). The black, blue and green profiles indicate data collected during run $A, B$ and $C$ respectively for which each technical replicate received different barcode. All isolates were analysed in duplicates within each run. The list of bacterial taxa matching given LCp is given in the table. 


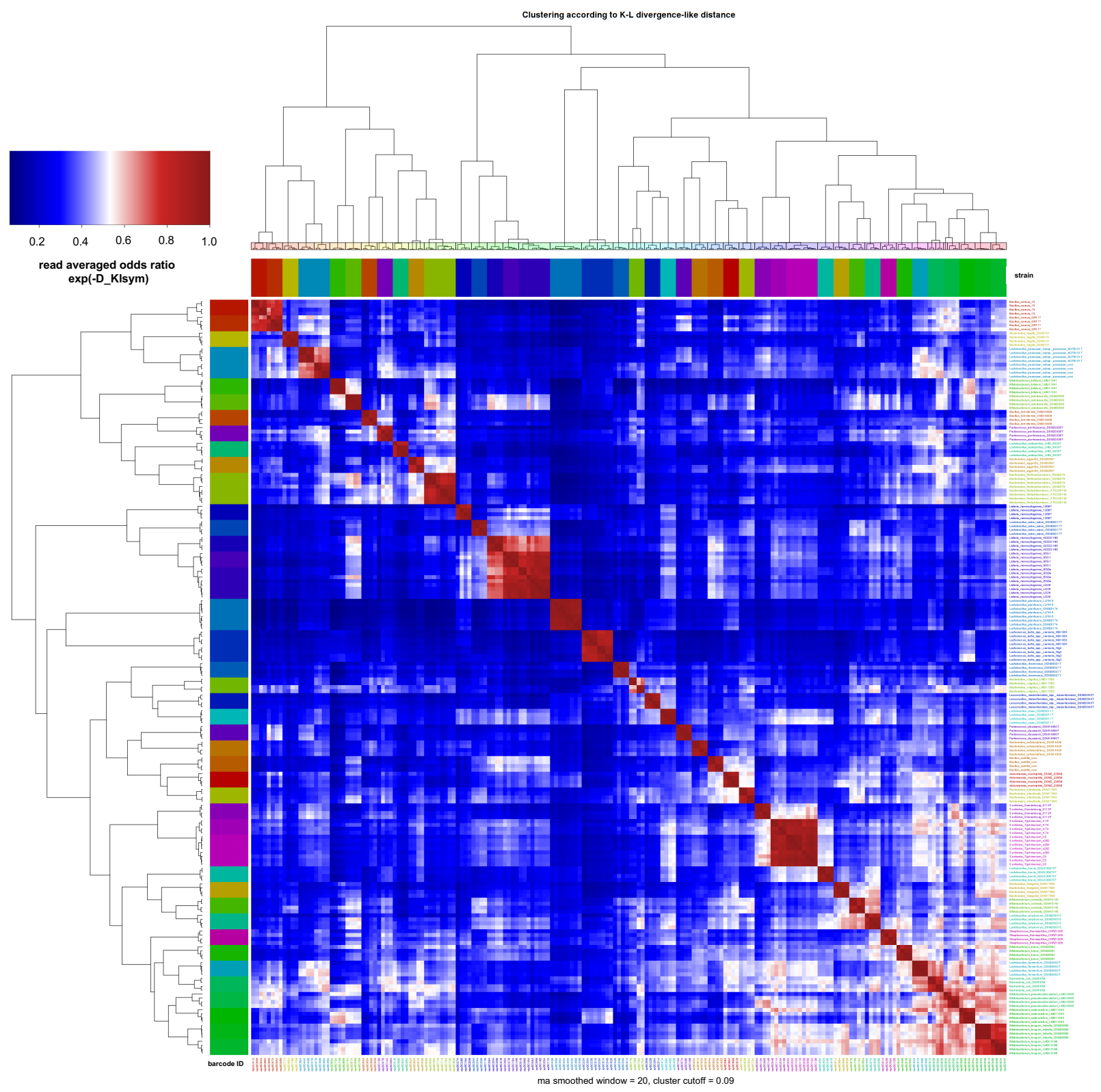

Supplementary Figure 2. Row/Column clustering according to "Ward.D2" hierarchical clustering on D_KLsym distance of all 48 isolates. Heatmap showing similarity $\left(10^{\wedge}\left(-D \_K L s y m\right)\right)$, and clustering according to cutoff $=0.09$. The detailed analysis using varying cutoff value (no single cutoff achieves exact separation between all and only different LCp, see Supplementary Figure $4 \mathrm{C}$, D ROC curves) and LCp visual inspection allowed for accurate differentiation between all except two pairs of bacterial strains described thoroughly in the results section (see Figure 3 and Supplementary figure 2 for details). Technical replicates from the third run "repC" were removed from the analysis due to higher short/long reads imbalance. 

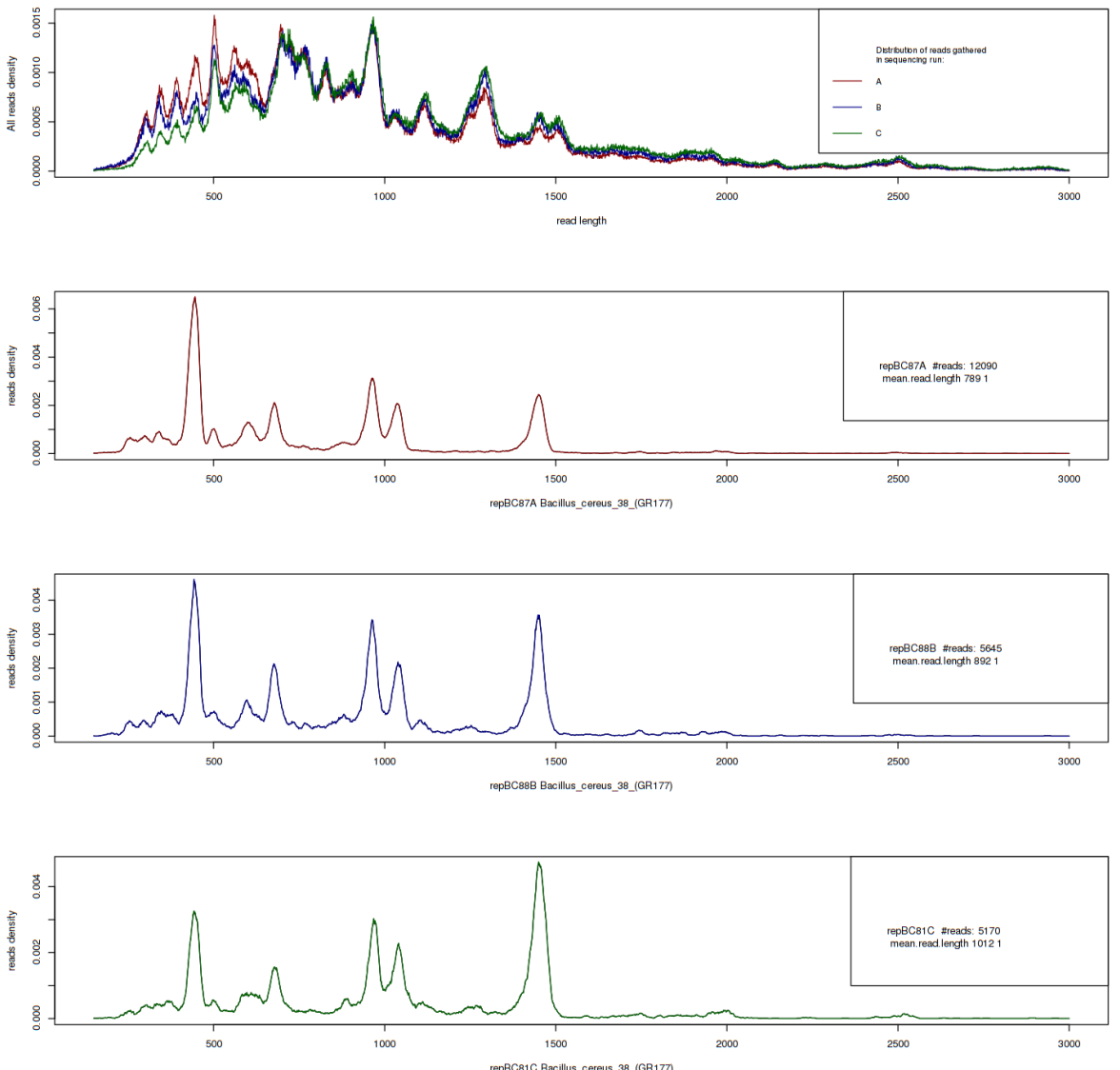

B

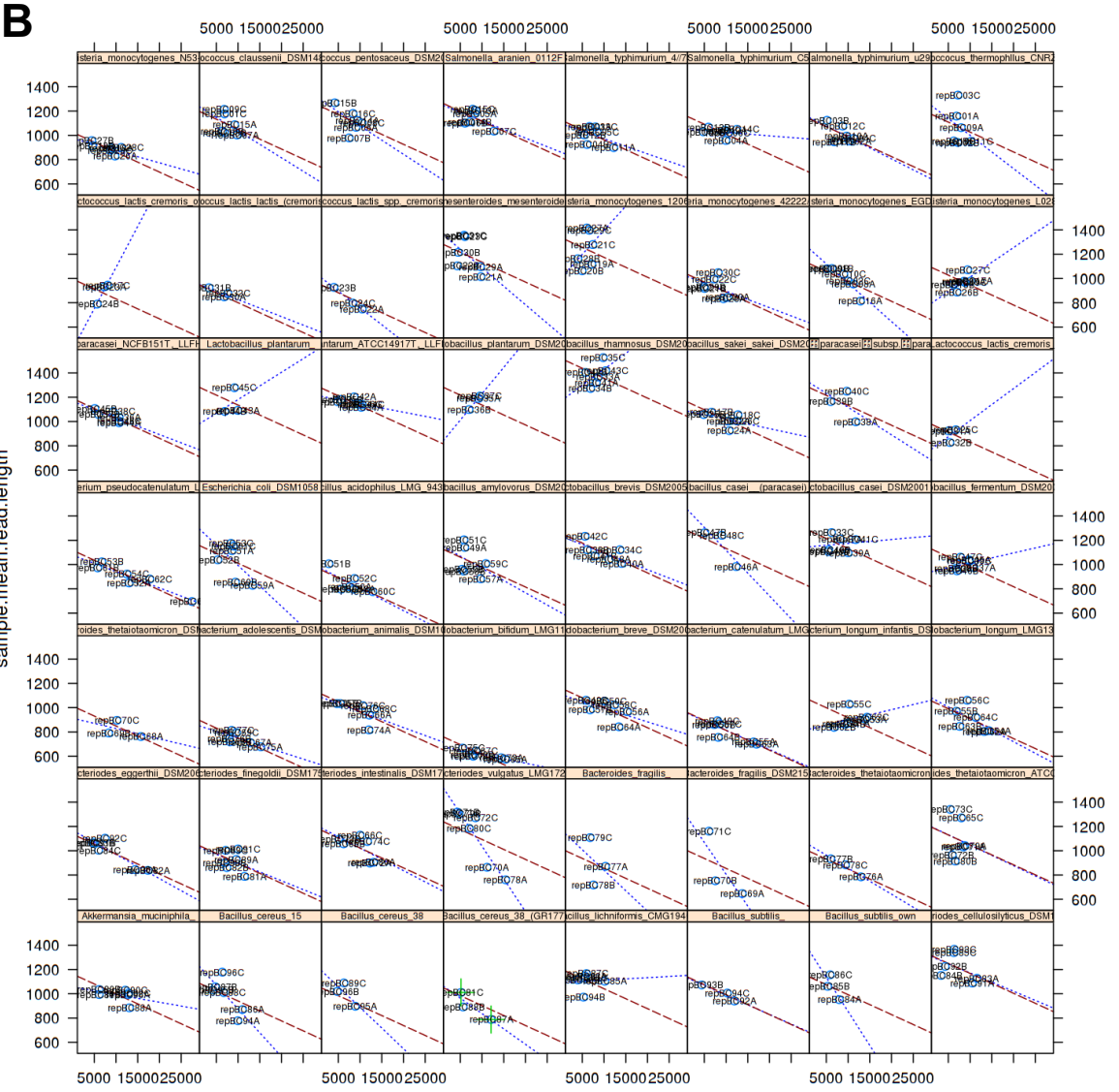

Supplementary Figure 3

A) Top panel presents distribution of lengths of reads obtained in 3 separate consecutive sequencing runs $A, B, C$ on the same flow cell. Third run $C$ obtained less short reads, some differences are also visible in second run $B$, compared to the first run A. Bottom 3 panels show LCps of

Bacillus cereus 38(GR177) strain obtained from runs $A, B, C$.

B) Regression analysis of mean read length from $L C p$ vs read count in LCp, data shown in separate panels for each strain replicates. Red dashed line is regression line obtained in all samples analysis, blue lines are regression lines for each strain only. Green markers mark runs $A$,

for Bacillus_cereus_38(GR177) (panels 2 and 4 in $\bar{A}$ ). 
A
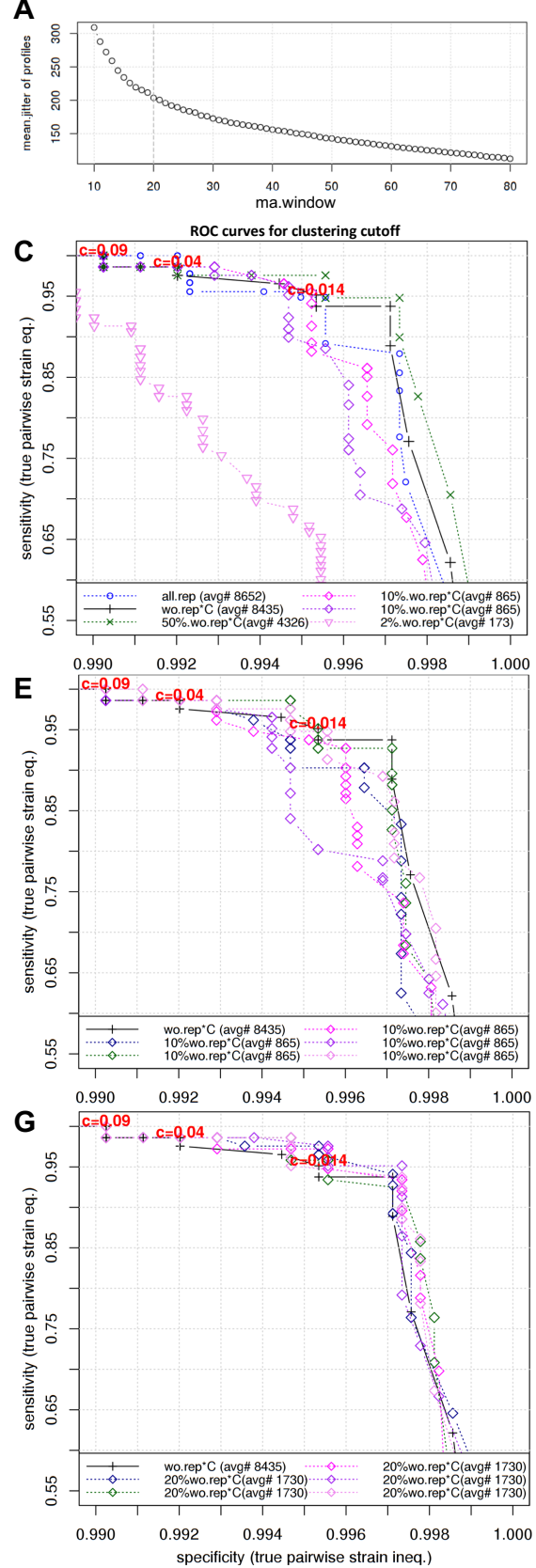
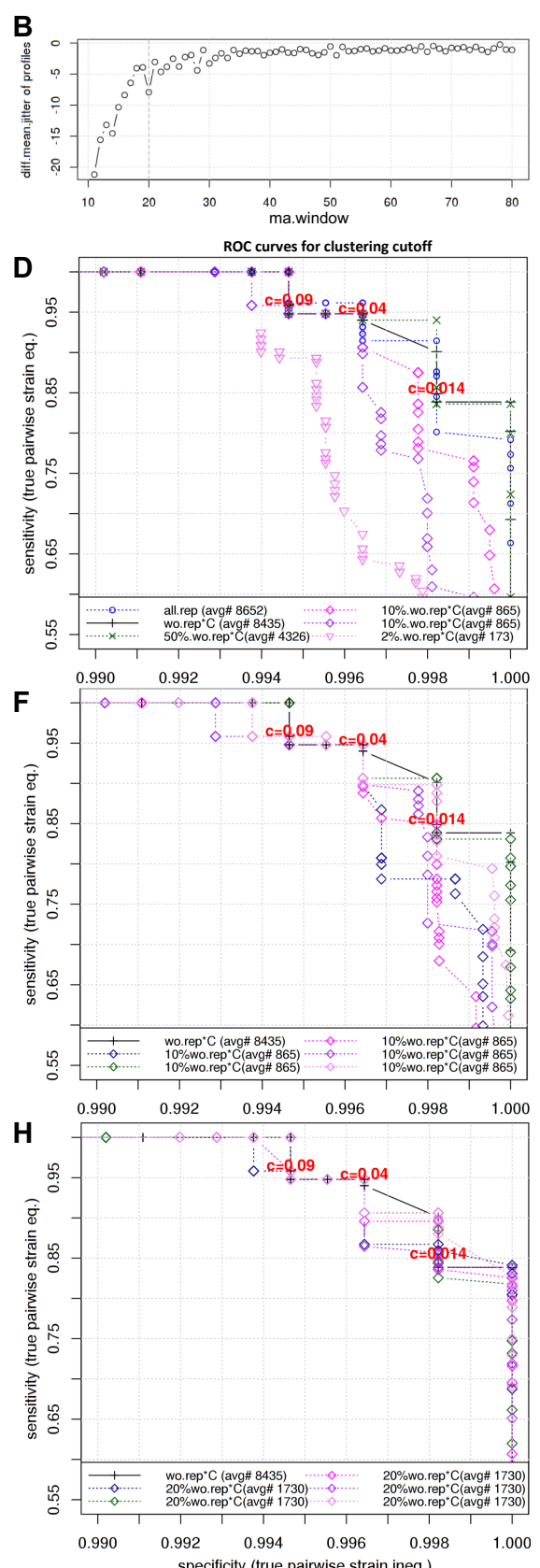

Supplementary Figure 4. Peaks profiles comparison

A) mean jitter of all profiles dependence on smoothing moving average "ma" window size. Jitter was defined as an average number of times when profile's discrete derivative changes sign (change to 0 was counted as 0.5 ). B) discrete derivative (diff lag=1) of the (top) mean jitter. Mean jitter changes more slowly and steadily with sizes of ma.window $>20$, suggestive of stabilization (noise decoupling) of information content in larger smoothing window results.

$\mathrm{C}-\mathrm{H})$ Receiver operating characteristic (ROC) curves of pairwise "same/not-thesame" strain discrimination in various cutoffs c (diff. step=0.005), for various subsets of data: "all", "wo.rep* $C^{*}$ dataset without the third sequencing run " $\mathrm{C}$ " on twice used flow cells, "50\%.wo.rep ${ }^{*} \mathrm{C}$ " subsample half the size of original, " $20 \%$.wo.rep ${ }^{*}$ " five subsamples $1 / 20^{\text {th }}$ of reads, $\quad 10 \%$.wo.rep ${ }^{*} \mathrm{C}$ seven subsamples $1 / 10^{\text {th }}$ of reads and " $2 \%$.wo.rep ${ }^{*} C " 1 / 50^{\text {th }}$ of reads. On $x-$ axis specificity, the percentage of correctly identified "not-the-same strain" pairs out of all such pairs (36096 for wo.rep ${ }^{*} C$ ), on $y$ axis sensitivity, the percentage of correctly identified "same strain" pairs, out of all such pairs (768 for wo.rep ${ }^{*} \mathrm{C}$ ). C) Clustering according to sample strain label, which can be thought of more as a whole method performance, in contrast to $D$. Some values on the plot: $c=0.09$ (sp 0.9902 , se 1.0), $c=0.014$ (sp 0.9953 , se 0.9514). D) Clustering according to sample strain similarity derived from visual inspection of profiles, thus these curves correspond more to D_KLsym-based profile comparison performance, than to the whole method. Values on the plot: $\mathrm{c}=0.09 \quad$ (sp $\quad 0.9947$, se 0.9583 ), $c=0.014$ (sp 0.9982, se 0.8490). All cutoffs "c" values marked for "wo.rep ${ }^{*} \mathrm{C}$ ". E) Clustering according to sample strain label using 5 iterations of $10 \%$ subsets. F) Clustering according to sample strain similarity derived from visual inspection of profiles using 5 iterations of $20 \%$ subsets. The analysis shows that $20 \%$ subsets perform similarly to the whole dataset what indicates the theoretical throughput of ON-rep-seq to range from 960 (if generating $\sim 1.5 \mathrm{M}$ reads) to 1440 (if generating $\sim 2.5 \mathrm{M}$ reads) isolates per flow cell. 
Supplementary Table 1. Identification of MLST genes alleles among selected strains of Salmonella enterica subsp. enterica serovar Typhimurium and Listeria monocytogenes

\begin{tabular}{|c|c|c|c|c|c|}
\hline Strain & Gene & Allele type & Strain & Gene & Allele type \\
\hline \multirow{7}{*}{$\begin{array}{c}\text { Salmonella enterica } \\
\text { U292 }\end{array}$} & aroC & 10 & \multirow{7}{*}{$\begin{array}{c}\text { Listeria } \\
\text { monocytogenes } \\
\text { EDGe }\end{array}$} & $a b c Z$ & 6 \\
\hline & dnaN & 7 & & bglA & 5 \\
\hline & hemD & 12 & & cat & 6 \\
\hline & hisD & 9 & & dapE & 20 \\
\hline & purE & 5 & & dat & 176 \\
\hline & sucA & 9 & & Idh & 4 \\
\hline & thrA & 2 & & Ihk & 1 \\
\hline \multirow{7}{*}{$\begin{array}{c}\text { Salmonella enterica } \\
\text { C5 }\end{array}$} & aroC & 10 & \multirow{7}{*}{$\begin{array}{c}\text { Listeria } \\
\text { monocytogenes } \\
\text { L028 }\end{array}$} & $a b c Z$ & 6 \\
\hline & dnaN & 7 & & bglA & 5 \\
\hline & hemD & 12 & & cat & 6 \\
\hline & hisD & 9 & & dapE & 51 \\
\hline & purE & 5 & & dat & 176 \\
\hline & sucA & 9 & & Idh & 4 \\
\hline & thrA & 2 & & lhk & 1 \\
\hline \multirow{7}{*}{$\begin{array}{c}\text { Salmonella enterica } \\
4 / 74\end{array}$} & aroC & 10 & & & \\
\hline & dnaN & 7 & & & \\
\hline & hemD & 12 & & & \\
\hline & hisD & 9 & & & \\
\hline & purE & 5 & & & \\
\hline & sucA & 9 & & & \\
\hline & thrA & 2 & & & \\
\hline
\end{tabular}


Supplementary Table 2. Details regarding benchmarking of two R9.4.1 flow cells

\begin{tabular}{|c|c|c|c|c|c|c|c|}
\hline \multirow[b]{2}{*}{ Run ID } & \multicolumn{4}{|c|}{ Flow cell 1} & \multicolumn{3}{|c|}{ Flow cell 2} \\
\hline & A & B & $\mathbf{C}$ & D & A & B & $\mathbf{C}$ \\
\hline Run Time (h) & 4 & 4 & 4 & 4 & 4 & 4 & 12 \\
\hline Break between the next run (day) & 1 & 4 & 3 & 7 & 1 & 1 & 1 \\
\hline Active pores at start & 1347 & 1324 & 1098 & 925 & 1034 & 779 & 615 \\
\hline Voltage at start (mV) & -180 & -180 & -190 & -195 & -180 & -180 & -190 \\
\hline Initial sequences in strand & $\sim 300$ & $\sim 200$ & $\sim 150$ & $\sim 50$ & $\sim 200$ & $\sim 120$ & $\sim 70$ \\
\hline Total number of high quality reads collected & $9.4 \cdot 10^{5}$ & $7.9 \cdot 10^{5}$ & $5.7 \cdot 10^{5}$ & $2.2 \cdot 10^{5}$ & $10.5 \cdot 10^{5}$ & $5.7 \cdot 10^{5}$ & $8.7 \cdot 10^{5}$ \\
\hline Library concentration loaded in $12 \mu \mathrm{l}(\mathrm{ng} / \mu \mathrm{l})$ & 2.5 & 1.8 & 3.0 & 1.6 & 3.2 & 2.1 & 2.4 \\
\hline
\end{tabular}

Both flow cells generated in total similar amount of data, although flow cell 1 was in much better condition and had more active pores at start what allowed to perform four consecutive runs. Flow cell 2 had lower number of active pores at arrival and seemed to deteriorate faster therefore only three runs were conducted. Last run was elongated to $12 \mathrm{~h}$ in order to collect maximum amount of data from declining flow cell. The data from the first benchmarked flow cell were used solely to test the optimal concentration of DNA needed and viability of the flow cell while data from the second flow cell are presented herein 
Supplementary Table 3. List of 96 barcodes for bacterial isolate Rep-PCR amplicon tagmentation. Rep-BC01 GTCTCGTCCGCTCGg CACAAAGACACCGACAACTTTCTT GTGGTGGTGGTGGTG Rep-BC02 GTTAGTTGATGTAGT ACAGACGACTACAAACGGAATCGA GTGGTGGTGGTGGTG Rep-BC03 GTCTCGTCCGCTCGG CCTGGTAACTGGGACACAAGACTC GTGGTGGTGGTGGTG Rep-BC04 GTTAGTTGATGTAGT TAGGGAAACACGATAGAATCCGAA GTGGTGGTGGTGGTG Rep-BC05 GTCTCGTCCGCTCGG AAGGTTACACAAACCCTGGACAAG GTGGTGGTGGTGGTG Rep-BC06 GTTAGTTGATGTAGT GACTACTTTCTGCCTTTGCGAGAA GTGGTGGTGGTGGTG Rep-BC07 GATATGATATAGATA AAGGATTCATTCCCACGGTAACAC GTGGTGGTGGTGGTG Rep-BC08 GTTAGTTGATGTAGT ACGTAACTTGGTTTGTTCCCTGAA GTGGTGGTGGTGGTG Rep-BC09 GTCTCGTCCGCTCGG AACCAAGACTCGCTGTGCCTAGTT GTGGTGGTGGTGGTG Rep-BC10 GTTAGTTGATGTAGT GAGAGGACAAAGGTTTCAACGCTT GTGGTGGTGGTGGTG Rep-BC11 GTTAGTTGATGTAGT TCCATTCCCTCCGATAGATGAAAC GTGGTGGTGGTGGTG Rep-BC12 GTCTCGTCCGCTCGG TCCGATTCTGCTTCTTTCTACCTG GTGGTGGTGGTGGTG Rep-BC13 GTCTCGTCCGCTCGG TCACACGAGTATGGAAGTCGTTCT GTGGTGGTGGTGGTG Rep-BC14 GTTAGTTGATGTAGT TCTATGGGTCCCAAGAGACTCGTT GTGGTGGTGGTGGTG Rep-BC15 GTTAGTTGATGTAGT CAGTGGTGTTAGCGAGGTAGACCT GTGGTGGTGGTGGTG Rep-BC16 TACATTGATGCATGG AGTACGAACCACTGTCAGTTGACG GTGGTGGTGGTGGTG Rep-BC17 GTCTCGTCCGCTCGG ATCAGAGGTACTTTCCTGGAGGGT GTGGTGGTGGTGGTG Rep-BC18 GTTAGTTGATGTAGT GCCTATCTAGGTTGTTGGGTTTGG GTGGTGGTGGTGGTG Rep-BC19 GTTAGTTGATGTAGT ATCTCTTGACACTGCACGAGGAAC GTGGTGGTGGTGGTG Rep-BC20 GTTAGTTGATGTAGT ATGAGTTCTCGTAACAGGACGCAA GTGGTGGTGGTGGTG Rep-BC21 GTTAGTTGATGTAGT TAGAGAACGGACAATGAGAGGCTC GTGGTGGTGGTGGTG Rep-BC22 GTTAGTTGATGTAGT CGTACTTTGATACATGGCAGTGGT GTGGTGGTGGTGGTG Rep-BC23 GTCTCGTCCGCTCGg CGAGGAGGTTCACTGGGTAGTAAG GTGGTGGTGGTGGTG Rep-BC24 GTTAGTTGATGTAGT CTAACCCATCATGCAGAACTATGC GTGGTGGTGGTGGTG Rep-BC25 GTCTCGTCCGCTCGG CATTGCGTTGCATACCCAACTTAC GTGGTGGTGGTGGTG Rep-BC26 TACATTGATGCATGG ATGAGAATGCGTAGTCGCTGTATG GTGGTGGTGGTGGTG Rep-BC27 GTCTCGTCCGCTCGG TGTAAGAGGTGAATCTAACCGTCG GTGGTGGTGGTGGTG Rep-BC28 GTTAGTTGATGTAGT GATACGGTGCCTTCTTAGGTTTCA GTGGTGGTGGTGGTG Rep-BC29 GTTAGTTGATGTAGT GGTCTGTCAACCCAAGGTGTCTAG GTGGTGGTGGTGGTG Rep-BC30 GTTAGTTGATGTAGT TGGGTCGAAGTAGATCCTCACTGA GTGGTGGTGGTGGTG Rep-BC31 GTCTCGTCCGCTCGG CAATGTAACTGATTGCTGTACGCA GTGGTGGTGGTGGTG Rep-BC32 GTTAGTTGATGTAGT ATGACGTTGTCGGACTTCTACTGG GTGGTGGTGGTGGTG Rep-BC33 GTCTCGTCCGCTCGG AGTTACCCAACCGTACCAAGTCTG GTGGTGGTGGTGGTG Rep-BC34 GTTAGTTGATGTAGT GCCTTTGACTTGAGTTCTTCGTCC GTGGTGGTGGTGGTG Rep-BC35 GTCTCGTCCGCTCGG GCAGTCCCTCAGCTTCGTAAGTAG GTGGTGGTGGTGGTG Rep-BC36 GTTAGTTGATGTAGT TGTTTCCTCCTCTAACTGGGACAT GTGGTGGTGGTGGTG Rep-BC37 GTCTCGTCCGCTCGG TGATACTAAGCATCAATCGCAAGC GTGGTGGTGGTGGTG Rep-BC38 GTTAGTTGATGTAGT TTCTCTGTATCGTCCTCCTGTGGT GTGGTGGTGGTGGTG Rep-BC39 GTTAGTTGATGTAGT GAGAGGCTCTAGTTGACACTGTGG GTGGTGGTGGTGGTG Rep-BC40 GTTAGTTGATGTAGT GGCTATCCTTGGTCATCCAAACTA GTGGTGGTGGTGGTG Rep-BC41 GTTAGTTGATGTAGT CGTGTACTTCTCTGGACGAACTCC GTGGTGGTGGTGGTG Rep-BC42 GTCTCGTCCGCTCGG CTGGCAGGTATGCCTTACACGTAG GTGGTGGTGGTGGTG Rep-BC43 GTTAGTTGATGTAGT CTACCGTCGAGTCAACAACGAAAG GTGGTGGTGGTGGTG Rep-BC44 GTTAGTTGATGTAGT GAGTGGGAAGGAACCCTTTCTACT GTGGTGGTGGTGGTG Rep-BC45 GTCTCGTCCGCTCGG CACTGAAGGCATCTCTGTTGGATC GTGGTGGTGGTGGTG Rep-BC46 GTTAGTTGATGTAGT CAGGAGAATGAAGTGGAACACAGC GTGGTGGTGGTGGTG Rep-BC47 GTCTCGTCCGCTCGG GAACTACCTGTGGGAAAGTTGCAC GTGGTGGTGGTGGTG Rep-BC48 GTTAGTTGATGTAGT TACAGGTGTACCACGTTCCAGATG GTGGTGGTGGTGGTG 
$\begin{array}{lll}\text { name spacer } & \text { ONbarcode } & \text { 5x(GTG) }\end{array}$

Rep-BC49 GTCTCGTCCGCTCGg CTAGATGTTCAAAGCTGCACCAGT GTGGTGGTGGTGGTG

Rep-BC50 GTTAGTTGATGTAGT ACGCAGGAAGTTACCAAAGTCCAT GTGGTGGTGGTGGTG

Rep-BC51 GTCTCGTCCGCTCGG GAGGACCCAGTAGGCTCATTCAAC GTGGTGGTGGTGGTG

Rep-BC52 GTTAGTTGATGTAGT GTCCACGAACAATCTTGTCTCTCA GTGGTGGTGGTGGTG

Rep-BC53 GTCTCGTCCGCTCGG CTTTGCATGAGACGGTCTGAATCT GTGGTGGTGGTGGTG

Rep-BC54 GTTAGTTGATGTAGT CATGCTCCTTAGTCAAAGCTCTTG GTGGTGGTGGTGGTG

Rep-BC55 GTCTCGTCCGCTCGG CGTAGATCAGGGTCTCATCTTCCA GTGGTGGTGGTGGTG

Rep-BC56 GTCTCGTCCGCTCGG TTCATGCCACCTGTTGAGTAGTGA GTGGTGGTGGTGGTG

Rep-BC57 TACATTGATGCATGG ACTTCCGAAGGAGATTGACCTAGC GTGGTGGTGGTGGTG

Rep-BC58 GTTAGTTGATGTAGT TCAGACTCACGGAGGAGTAACCTG GTGGTGGTGGTGGTG

Rep-BC59 GTTAGTTGATGTAGT ACCTTGCTTTCCCTTCTTGATTGA GTGGTGGTGGTGGTG

Rep-BC60 GTTAGTTGATGTAGT CCATAGAAGCCTTGGTTGAACATG GTGGTGGTGGTGGTG

Rep-BC61 TACATTGATGCATGG GTGCTGAGGCACATAGTACCCTCT GTGGTGGTGGTGGTG

Rep-BC62 GTTAGTTGATGTAGT TACGTCCTGAAGTAAGTGTGGGTG GTGGTGGTGGTGGTG

Rep-BC63 GTTAGTTGATGTAGT GTTCAAGACCCAGGAACTTCAGAA GTGGTGGTGGTGGTG

Rep-BC64 GTTAGTTGATGTAGT GAAAGTCGATGAACGGTGTCTGTC GTGGTGGTGGTGGTG

Rep-BC65 GTCTCGTCCGCTCGg CCTTGTCTGGAGGAAGACTGAGAA GTGGTGGTGGTGGTG

Rep-BC66 GTCTCGTCCGCTCGg GAAGTTAGAAGCCACAAGGATCGG GTGGTGGTGGTGGTG

Rep-BC67 TACATTGATGCATGG GGTGAGCACACGAGTATGACAAAC GTGGTGGTGGTGGTG

Rep-BC68 GTCTCGTCCGCTCGG CCACCTTCGTGTTTGCTTAGATTC GTGGTGGTGGTGGTG

Rep-BC69 GTTAGTTGATGTAGT AGATCACATGAGGCTCGGACTGTA GTGGTGGTGGTGGTG

Rep-BC70 GTTAGTTGATGTAGT ACACTCCATTCGTAGGATCTCGGT GTGGTGGTGGTGGTG

Rep-BC71 GTCTCGTCCGCTCGg CTGTTACTACCTGATGCTCCCAGG GTGGTGGTGGTGGTG

Rep-BC72 GTTAGTTGATGTAGT GTCGGTATGGAAGACAGTCAGCTA GTGGTGGTGGTGGTG

Rep-BC73 GTCTCGTCCGCTCGG GAGGGTTCTGTCATCCTGTTTCTT GTGGTGGTGGTGGTG

Rep-BC74 GTTAGTTGATGTAGT AGTGGAAGTGTTGGGATGCTTGTA GTGGTGGTGGTGGTG

Rep-BC75 GTCTCGTCCGCTCGG ACAACAGGGTTCATCACAATGGTC GTGGTGGTGGTGGTG

Rep-BC76 GTTAGTTGATGTAGT GTCCAGGGTTGATGTAACAAGCAT GTGGTGGTGGTGGTG

Rep-BC77 GTCTCGTCCGCTCGg GTTGTATCCCTGAGAAACAGGTCG GTGGTGGTGGTGGTG

Rep-BC78 GTTAGTTGATGTAGT TTCTGATTCAAAGGTTCGGTTGTT GTGGTGGTGGTGGTG

Rep-BC79 GTCTCGTCCGCTCGG CAGCAGTGAGAACTATCTCCGAGA GTGGTGGTGGTGGTG

Rep-BC80 GTTAGTTGATGTAGT GAATCGCTATCCTATGTTCATCCG GTGGTGGTGGTGGTG

Rep-BC81 GTCTCGTCCGCTCGg CCGAAACAACTTCACAAGATGAGG GTGGTGGTGGTGGTG

Rep-BC82 GTTAGTTGATGTAGT TAGTCCTGGAACTCGACATACCGT GTGGTGGTGGTGGTG

Rep-BC83 GTCTCGTCCGCTCGG TTCGACCTTACCTAGATCAAGCCA GTGGTGGTGGTGGTG

Rep-BC84 GTTAGTTGATGTAGT TGGCACAGGTTCTAGGTCCACTAC GTGGTGGTGGTGGTG

Rep-BC85 GTCTCGTCCGCTCGg GATCATCCAACTAACTCCTCCGTT GTGGTGGTGGTGGTG

Rep-BC86 GTCTCGTCCGCTCGG TACTTACGCTTGTTGGGATCACCT GTGGTGGTGGTGGTG

Rep-BC87 GTCTCGTCCGCTCGG CCTCCCTAACAACAGGAGCATGTA GTGGTGGTGGTGGTG

Rep-BC88 GTTAGTTGATGTAGT CTGCTTCGGATCGGTAGTAGAAGA GTGGTGGTGGTGGTG

Rep-BC89 GTCTCGTCCGCTCGG CAACTAGCCAAACATTGATGCTGT GTGGTGGTGGTGGTG

Rep-BC90 GTTAGTTGATGTAGT GCCTCAAACCGTACCCTCTACATC GTGGTGGTGGTGGTG

Rep-BC91 GTCTCGTCCGCTCGG AGTAGCGTGAGTTCCTATGGAGCC GTGGTGGTGGTGGTG

Rep-BC92 GTCTCGTCCGCTCGg GGTCCTGTATCTTTCCACTCACAA GTGGTGGTGGTGGTG

Rep-BC93 GTCTCGTCCGCTCGG CCCAAGTCTGAAGTGATGGAAACT GTGGTGGTGGTGGTG

Rep-BC94 GTTAGTTGATGTAGT GTAGGTGGCAGTTTGAGGACAATC GTGGTGGTGGTGGTG

Rep-BC95 GTCTCGTCCGCTCGG AAGTCCATTCTTCTTCCAGACAGG GTGGTGGTGGTGGTG

Rep-BC96 GTCTCGTCCGCTCGG ATGGTGGACTCTATGACCGTTCAG GTGGTGGTGGTGGTG 